ARTICLE

\title{
Positron emission tomography imaging of novel AAV capsids maps rapid brain accumulation
}

Jai Woong Seo (1) 1,2, Elizabeth S. Ingham², Lisa Mahakian², Spencer Tumbale¹, Bo Wu1, Sadaf Aghevlian', Shahin Shams (10 2, Mo Baikoghli ${ }^{3}$, Poorva Jain ${ }^{1}$, Xiaozhe Ding (10 ${ }^{4}$, Nick Goeden ${ }^{4}$, Tatyana Dobreva ${ }^{4}$, Nicholas C. Flytzanis (1) 4, Michael Chavez ${ }^{5}$, Kratika Singhal ${ }^{6}$, Ryan Leib (i) ${ }^{6}$, Michelle L. James ${ }^{1}$, David J. Segal (10 ${ }^{7}$, R. Holland Cheng (1) ${ }^{3}$, Eduardo A. Silva (1) ${ }^{2}$, Viviana Gradinaru (iD ${ }^{4} \&$ Katherine W. Ferrara (iD ${ }^{1 \times}$

Adeno-associated viruses (AAVs) are typically single-stranded deoxyribonucleic acid (ssDNA) encapsulated within 25-nm protein capsids. Recently, tissue-specific AAV capsids (e.g. PHP.eB) have been shown to enhance brain delivery in rodents via the LY6A receptor on brain endothelial cells. Here, we create a non-invasive positron emission tomography (PET) methodology to track viruses. To provide the sensitivity required to track AAVs injected at picomolar levels, a unique multichelator construct labeled with a positron emitter (Cu-64, $t_{1 / 2}=12.7 \mathrm{~h}$ ) is coupled to the viral capsid. We find that brain accumulation of the PHP.eB capsid 1) exceeds that reported in any previous PET study of brain uptake of targeted therapies and 2) is correlated with optical reporter gene transduction of the brain. The PHP. eB capsid brain endothelial receptor affinity is nearly 20 -fold greater than that of AAV9. The results suggest that novel PET imaging techniques can be applied to inform and optimize capsid design.

\footnotetext{
${ }^{1}$ Molecular Imaging Program at Stanford (MIPS), Department of Radiology, School of Medicine, Stanford University, Stanford, CA, USA. ${ }^{2}$ Department of Biomedical Engineering, University of California, Davis, CA, USA. ${ }^{3}$ Department of Molecular and Cellular Biology, University of California, Davis, CA, USA. ${ }^{4}$ Division of Biology and Biological Engineering, California Institute of Technology, Pasadena, CA, USA. ${ }^{5}$ Department of Bioengineering, Stanford University, Stanford, CA, USA. ${ }^{6}$ Stanford University Mass Spectrometry, Stanford, CA, USA. ${ }^{7}$ Genome Center and Department of Biochemistry and Molecular Medicine, University of California, Davis, CA, USA. ${ }^{{ }}$email: kwferrar@stanford.edu
} 
T herapeutic delivery to the brain has traditionally been limited in volume. The background level of protein/ nanotherapeutics reaching the brain is on the order of 0.1 percent injected dose per cubic centimeter $(\% \mathrm{ID} / \mathrm{cc})^{1}$, necessitating more efficient methods of delivery. Engineered adenoassociated viruses (AAVs), single-stranded deoxyribonucleic acid (ssDNA) encapsulated within $25-\mathrm{nm}$ protein capsids, have recently shown potential to greatly increase transduction as compared with previous therapeutics ${ }^{2-4}$. AAVs can infect dividing and non-dividing cells and result in highly efficient long-term transduction in a broad range of tissues 5,6 . This is particularly significant as AAV gene therapy has a solid safety profile, was first approved by the FDA in December $2017^{7}$ and more than 200 clinical trials have been conducted since $1989^{8}$. Recently, AAVs have been shown capable of delivering CRISPR-Cas9 gene silencing in vivo ${ }^{9}$, expanding their potential utility. Using a directed evolution approach to viral capsid engineering and selection, AAV-PHP.eB, containing a 2-mer substitution and 7mer peptide insertion in a surface exposed loop of the capsid, enhanced neuronal transduction throughout the brain compared to the conventionally used AAV serotype 9 (AAV9) ${ }^{3}$. This 40 to 90 -fold increased efficiency is believed to result from a novel interaction between virus and the brain endothelial cell receptor LY6A $^{10,11 .}$.

In vivo imaging has great potential to contribute to the design and optimization of AAVs. The biodistribution of viral vectors has previously been evaluated by real-time PCR, Southern blotting of the transduced gene, western blotting, immunohistochemistry (IHC), and in vivo imaging of reporter proteins ${ }^{12}$. Many of these methods are invasive, relying on small quantities of tissue at a single site and/or time point ${ }^{13}$. In vivo imaging can determine the reporter protein level expressed from a transduced gene across an entire region of interest (ROI) over time; however, the underlying mechanisms for differences in the reporter protein cannot be directly identified with this approach. Development of a labeling method for non-invasive pharmacokinetics (PK) studies is valuable for several reasons. First, in vivo imaging can directly and non-invasively assess endothelial receptor binding at multiple time points. Second, PK can be non-invasively assessed even with repeated administration, the potential for which increases since capsid engineering and cargo development also address issues related to AAV neutralization and immunogenicity $5,14,15$. Third, quantitative imaging techniques facilitate comparisons across strains and species.

We therefore set out to develop an imaging method to track therapeutic viral constructs and quantify their binding to endothelial surface receptors. Positron emission tomography (PET) provides an ideal non-invasive method to track viral constructs in brain-related and other diseases ${ }^{16}$. In particular graphical analysis of plasma and tissue radiotracer uptake at multiple time points produces a linear plot, the slope of which is related to the number of available tracer binding sites. PET facilitates the interpretation of endothelial binding and the quantification of reversible receptor binding 17,18 . This provides a unique noninvasive assessment of AAV uptake.

PET imaging has not previously been applied for systemic AAV tracking. Surface modification of AAVs has previously focused on tagging fluorophores ${ }^{19-24}$ to $\mathrm{PEG}^{25,26}$, or adding peptides $^{27,28}$, antibodies ${ }^{23}$, or small molecules ${ }^{29}$ to the capsid. Given that most earlier generations of $\mathrm{AAV}$ and other viral therapies were not designed for specific organ targeting, imaging studies labeled multiple lysines on the capsid with a lesser impact on organ-specific endothelial targeting and transduction ${ }^{30}$. Recently, the AAV capsid was labeled with I-124, but the study was limited to direct intracranial injection to the brain and therefore did not focus on the receptor binding characteristics or endothelial accumulation ${ }^{31}$. Alternatively, reporter gene imaging has been used to measure transduction but cannot quantify $\mathrm{PK}^{32}$. Thus, our study fills a void in PET imaging of the PK of novel capsids.

The challenge in monitoring the PK of systemically injected AAVs with PET (particularly with high time resolution) is to achieve a trackable level of radioactivity while matching the halflife of the positron emitter to AAV circulation half-life, which ranges from minutes to days ${ }^{33}$. An additional challenge is to minimize conjugation to key AAV surface features. High molar activity (MA) positron emitters, such as F-18 and Ga-68, typically have a short half-life ( $t_{1 / 2}$ of 110 and $68 \mathrm{~min}$, respectively); thus, limiting their utility (Fig. 1a). The dose for systemic administration of AAVs in mice is low; $~ 10^{11-12}$ vector genomes (vg) are injected, corresponding to $0.2-2$ pmol of AAVs. Cu-64 has a halflife of $12.7 \mathrm{~h}$ and is therefore well suited to the AAV half-life in blood ${ }^{34}$; however, combining ${ }^{64} \mathrm{CuCl}_{2}(\mathrm{MA}, \sim 20 \mu \mathrm{Ci} / \mathrm{pmol})$ and 2 pmol of AAVs yields $\sim 40 \mu \mathrm{Ci}$ of labeled AAVs when the labeling ratio of $\mathrm{Cu}-64$ to AAVs is 1:1. Real-time high resolution imaging is impaired with this very low level of radioactivity. In order to facilitate high signal-to-noise (SNR) PET imaging at a low AAV dose, we have therefore synthesized a bifunctional multichelator that increases the MA of ${ }^{64} \mathrm{Cu} /$ molecule up to 10 times compared to a single chelator.

Our study highlights the potential to use PET imaging to track viral capsids after systemic injection, facilitating noninvasive quantitation of organ accumulation and clearance and endothelial receptor binding. The multichelator approach developed here is applied for optical microscopy, system-level PET imaging and autoradiography. Based on these analyses, we find that brain accumulation of PHP.eB, a novel AAV9 derivative with high brain tropism, exceeds that reported in previous PET studies of brain uptake of targeted therapies. Further, the high signal-tonoise ratio obtained with the multichelator approach can be exploited to quantify endothelial receptor affinity over the first 30 min after injection. Here, brain affinity of the PHP.eB capsid is enhanced nearly 20 -fold as compared with the well-established AAV9 capsid. Most importantly, the labeling method retains the transduction efficacy of the AAV and can be applied in future studies to inform and optimize the design of AAVs and other viral capsids.

\section{Results}

Syntheses of multichelators. We have developed a bioorthogonal approach for coupling a multichelator and AAV, based on conjugation to AAV surface lysines and cysteines and used this approach to compare the PK of AAV9-PHP.eB (AAV9PHP.eB is denoted as PHP.eB hereafter) with AAV9 and AAV9tetracysteine (AAV9-TC). Conjugation to surface lysines was previously shown to be feasible in fluorescence imaging where AAV surface lysines were modified with a fluorescent dye, which facilitated AAV tracking without hampering transduction efficiency ${ }^{19-22,35}$. Based on surface solvent accessibility in the X-ray structure of the AAV9 capsid $^{30}$, the estimated number of exposed lysines on AAV9 and PHP.eB ranges from 420 to 480 out of 1185 and 1245 total lysines, respectively (Fig. 1b). This includes 7-8 lysines per viral protein (VP), with one viral particle composed of 60 units of VP. We based the surface lysine labeling strategies on inverse electron demand Diels-Alder reactions (IEDDA), which offer a fast, quantitative $\left(>50,000 \mathrm{M}^{-1} \mathrm{~S}^{-1}\right)$ orthogonal reaction ${ }^{36}$. We modified a small number of the surface lysines with Tz-NHS ester, followed by conjugation of the ${ }^{64} \mathrm{Cu}$-multichelatortranscyclooctene (TCO), (NOTA) $)_{8}$-TCO (Fig. 1c). Multichelator-maleimide, (NOTA) ${ }_{8}-\mathrm{MI}$, was employed (Fig. 1d) to label AAV9-TC. Notation describing the labeled AAVs (e.g. 


\begin{tabular}{|lll|}
\hline a & $\begin{array}{l}\text { Vector } \\
\text { genome }(\mathbf{V g})\end{array}$ & $\begin{array}{l}\text { Vector genome } \\
(\mathbf{p m o l})\end{array}$ \\
\hline AAV & $1 \times 10^{11-12}$ & $0.2-2$ \\
\hline $\begin{array}{l}\text { PET } \\
\text { isotopes }\end{array}$ & Half-life & $\begin{array}{l}\text { Molar activity } \\
(\mathrm{MA}, \mu \mathrm{Ci} / \mathrm{pmol})\end{array}$ \\
\hline F-18 & $110 \mathrm{~min}$ & $50-100$ \\
\hline Ga-68 & $68.1 \mathrm{~min}$ & $>27$ \\
\hline Cu-64* & $12.7 \mathrm{~h}$ & $\sim 20$ \\
\hline $\mathrm{I}-124$ & 4.18 days & $\sim 27$ \\
\hline
\end{tabular}

b

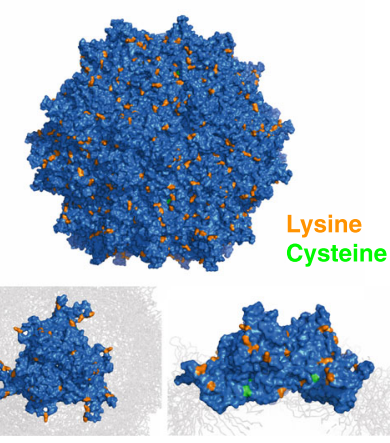

c

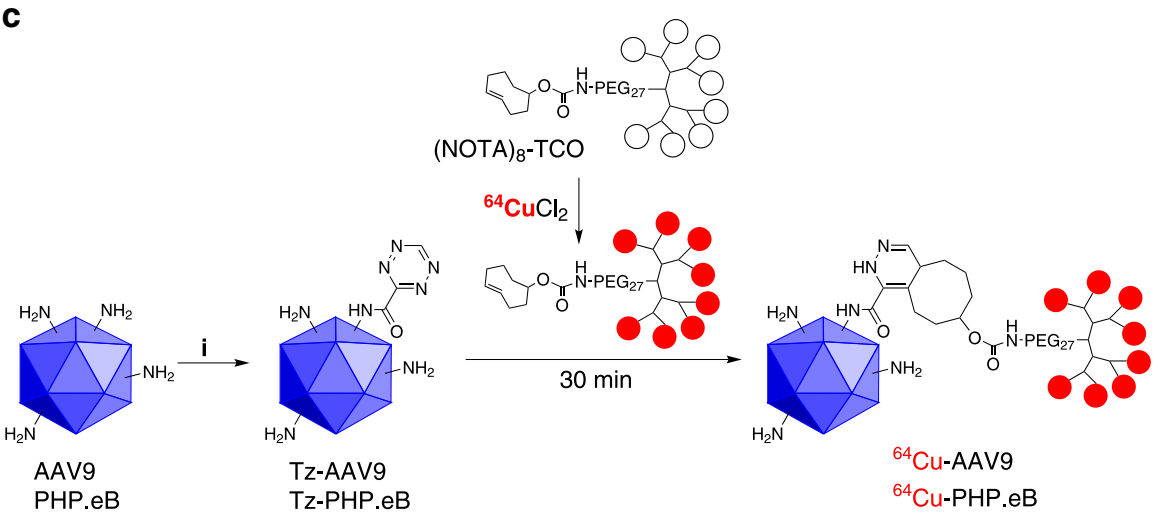

d

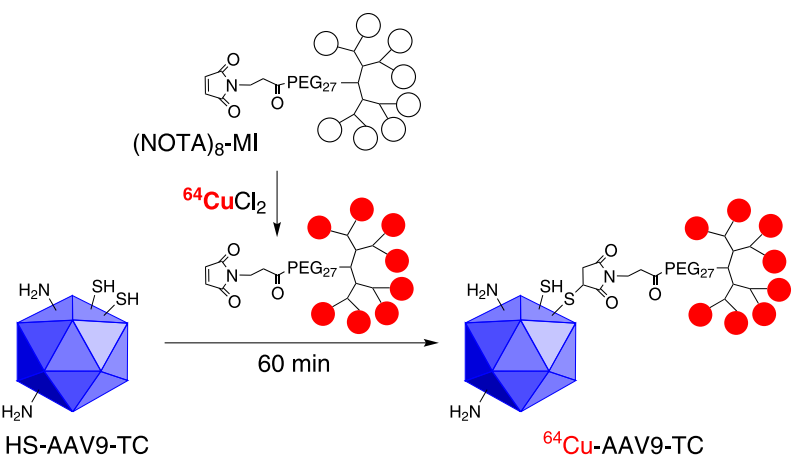

Fig. 1 Strategy for labeling AAVs with a positron emitter. a Table presents the number of AAVs systemically injected and the molar activity of positron emitters. b Solvent accessible surface of AAV9 capsid 30 (PDB ID:3UX1) displayed by PyMOL software. Insets highlight a trimer around a threefold axis. Orange and green color represent lysine and cysteine residues, respectively. Solvent radius is set as 1.4 angstrom. AAV capsid is composed of 60 structurally identical viral protein subunits (VPs) with 1:1:10 ratio of VP1:VP2:VP3. c, d are the labeling schemes of AAV-PHP.eB, AAV9, and AAV9-TC. c Surface modification with multichelators (MC) on lysine residues in capsids. (NOTA) ${ }_{8}$-TCO (incorporating a PEG 27 spacer) is employed for the radiolabeling of Tz-AAV9 or Tz-PHP.eB after reaction of Tz-NHS ester with AAV9 or PHP.eB. (i, tetrazine-PEG $5^{-N H S ~(T z-N H S) ~ e s t e r, ~} 1 x$ PBS (pH 8), $4{ }^{\circ} \mathrm{C}$, overnight dialysis in $20 \mathrm{kDa}$ molecular weight cut-off (MWCO) membrane). d The site-specific radiolabeling on cysteine residues in AAV9-TC was employed with the multichelator-maleimide conjugate ((NOTA) $)_{8}-\mathrm{MI}$ ) incorporating Cu-64 after the reduction of tetracysteine with TCEP (ii, TCEP in 1X PBS ( $\mathrm{pH}$ 7.0-7.5)). Asterisk indicates average molar radioactivity of $\mathrm{Cu}-64$ from commercial vendor as used in this study.

$\left.{ }^{64} \mathrm{Cu}-\mathrm{PHP} . \mathrm{eB}\right)$ is defined in Fig. 1c, d. In order to confirm that the lysine attachment does not alter receptor binding, we compared labeling with this approach to labeling of cysteines available on a tetracysteine (TC) engineered version of AAV9 using a maleimide-thiol reaction of the multichelator (Supplementary Fig. 1). In this virus, an (HRWCCPGCCKTF) motif was inserted at amino acid 139 (residue numbered by VP1 sequence) of the AAV9 capsid proteins (AAV9-139). The insertion site is located in the N-terminal disordered region of capsid proteins VP1 and VP2. A similar modification was made in AAV9 and was shown to be accessible by labeling reagents without compromising viral integrity ${ }^{24}$.

The multichelators, $(\mathrm{NOTA})_{8}$-TCO and $(\mathrm{NOTA})_{8}-\mathrm{MI}$, were synthesized through a solid phase reaction. Multistep coupling of
Fmoc-Lys(Fmoc)-OH from polyethylene glycol(PEG) ${ }_{27}$-Lys(Boc) on resin afforded eight branched amines, further coupled with tert-Bu-NOTA-OH. A PEG spacer was included to separate the multichelator and reactive functional group. After cleavage of (NOTA) ${ }_{8}-\mathrm{NH}_{2}$ (Supplementary Fig. 2a, 1) from the resin, 1 was further functionalized to (NOTA) ${ }_{8}$-TCO (Supplementary Fig. 2a, 2) and (NOTA) $)_{8}$-MI (Supplementary Fig. 2a, 3). In all, 2 and 3 were isolated by HPLC presented monoisotopic mass peaks at 5026.67 (calculated mass: 5026.85 Da) and 4937.66 (calculated mass: $4937.74 \mathrm{Da}$ ) in MALDI mass analysis (Supplementary Fig. 2a), respectively. For optical studies of PHP.eB and AAV9 conjugated with the multichelator, (NOTA) $)_{8}-\mathrm{A} 555-\mathrm{TCO}$ with a cysteine introduced to conjugate A555-maleimide was synthesized as shown in Supplementary Fig. $2 \mathrm{~b}$. The mass $\left(\mathrm{M}+\mathrm{H}^{+}\right)$of 
$(\mathrm{NOTA})_{8}-\mathrm{cys}(\mathrm{SH})-\mathrm{NH}_{2} \quad(4), \quad(\mathrm{NOTA})_{8}-\mathrm{A} 555-\mathrm{NH}_{2} \quad$ (5) and $(\mathrm{NOTA})_{8}$-A555-TCO (6) verified with MALDI mass analysis were detected at 4874.54 (calculated mass: $4875.70 \mathrm{Da}$ ), 5844.85 (calculated mass: 5843.94) and 6245.47 (calculated mass: 6243.16), respectively.

Efficiency of Cu-64 incorporation on single and multichelator. To assess the incorporation of copper on the multichelator, increasing amounts of (NOTA $)_{8}$-TCO were reacted with a known amount of nonradioactive $\mathrm{Cu}-63$ spiked with the radioactive $\mathrm{Cu}$ 64 , and the incorporation ratio was then compared with that achieved with the single chelator (NOTA-TCO). We confirmed that more than two single chelators, NOTA-TCOs, are required to incorporate one copper molecule (Supplementary Fig. 3a), and one multichelator, (NOTA) $)_{8}$ TCO, incorporated 5-8 copper molecules (Supplementary Fig. 3b). Thus, the multichelator achieves $\sim 10$ times higher molar radioactivity than the single chelator (Supplementary Fig. 3).

Capsid surface modification maintains transduction efficiency. To determine the maximum molar ratio of $\mathrm{Tz}-\mathrm{NHS}$ and (NOTA) ${ }_{8}$-TCO that can be incubated with PHP.eB:CAG-GFP (and similarly (NOTA) - $_{8}$ MI with AAV9-TC:CAG-mNeonGreen) without hampering its integrity, we monitored the AAV transduction efficiency in HEK293T cells before and after labeling (Fig. 2a, b, Supplementary Figs. 4 and 5). In previous studies, the conjugation of NHS (succinimidyl ester) to AAVs typically proceeded under strong basic conditions ( $\left.0.1 \mathrm{M} \mathrm{Na}_{2} \mathrm{CO}_{3}, \mathrm{pH} 9.3\right)$; however, the reported procedures have been inconsistently detailed, and the experimental conditions vary widely (summarized in Supplementary Table 1). To avoid harsh conditions, the reaction was performed at $\mathrm{pH} 8$ by mixing $\mathrm{PBS}$ and $\mathrm{Na}_{2} \mathrm{CO}_{3}$ (v:v, $8: 2$ ), as is routinely exploited in preparation of antibody conjugates. Under this reaction condition, PHP.eB $\left(4.2 \times 10^{11} \mathrm{vg}, 0.7\right.$ pmol particles) was incubated with Tz-NHS and followed an IEDDA reaction with (NOTA) ${ }_{8}$-TCO (Supplementary Fig. 4a). SDS-PAGE analysis of PHP.eBs labeled with (NOTA) - $^{-T C O}$ clearly showed three VP bands with similar molecular weight to the unmodified control PHP.eB (Supplementary Fig. 4b). Incubation with a molar ratio of 500 and above resulted in additional high molecular-weight protein bands (Supplementary Fig. 4b) and a significant reduction in fluorescent-protein expressing cells (Supplementary Fig. 4c). Both assays suggest that keeping the molar ratio of (NOTA) s $_{8}$ TCO/PHP.eB below 500-fold maintains transduction efficiency of HEK293T cells and prevents the aggregation of capsid proteins after labeling of PHP.eB. Limits on the concentration of the chelator were more restrictive with AAV9-TC. AAV9-TC:CAG-mNeonGreen $\left(5.8 \times 10^{12} \mathrm{vg}, \quad 9.6\right.$ pmol) after reduction to HS-AAV9-TC by TCEP was reacted with (NOTA) $)_{8}$-MIs at 14,70 , and 140 pmol (Supplementary Fig. 5a). Multiple bands of over-labeled VPs were found when the incubated (NOTA) ${ }_{8}$-MI/AAV9-TC ratio was 70 -fold or more (Supplementary Fig. 5b). For AAV9-TC, multiple bands likely result from the non-specific maleimide conjugation with primary amines as previously reported ${ }^{37}$. In this previous report, a similar protein band shift occurred in SDS-PAGE at dye:protein ratios $>40: 1$. Irrespective of the multiple band formation, AAV9-TC transduction efficiency was preserved at all levels of modification (Supplementary Fig. 5c).

Under the optimized conditions, AAV9 and PHP.eB were then labeled with multichelators that have been conjugated with $\mathrm{Cu}-64$ following the procedure detailed in the Methods section. Transduction efficiencies of Tz-AAV9 and -PHP.eB (modified from AAV9:CAG-mNeonGreen and PHP.eB:CAG-GFP with TzNHS, respectively) and HS-AAV9-TC (a reduced form of AAV9-
TC:CAG-mNeonGreen) were then compared with those of intact AAVs in HEK293T cells in vitro $\left(1 \times 10^{6} \mathrm{vg} / \mathrm{cell}\right)$. As assessed by fluorescent green protein expression in HEK293T cell images, fluorescent-protein transduction was similar at $24 \mathrm{~h}$ after incubation with intermediates before and after modification (Fig. 2a). Flow cytometry at $48 \mathrm{~h}$ confirmed the comparable transduction efficiency of modified and unmodified AAVs for TzAAV9 and -PHP.eB and HS-AAV9-TC (Fig. 2b). Most importantly, the transduction efficiency of ${ }^{63} \mathrm{Cu}$-labeled PHP. eB:CAG-GFP and PHP.eB:CAG-GFP in C57BL/6 mice was evaluated to determine whether the multichelator influenced viral delivery and GFP production in the brain at 3 weeks after tail vein administration $\left(1.5 \times 10^{10} \mathrm{vg}\right)$. The GFP mean fluorescence intensity (MFI) within the brain was similar (Fig. $2 c$, d, $n=$ 4 , n.s.) following injection of the unmodified (PHP.eB) or the labeled AAV ( $\left.{ }^{63} \mathrm{Cu}-\mathrm{PHP} . \mathrm{eB}\right)$ and undetectable after saline injection. Taken together, the results demonstrate that our optimized labeling condition preserved the AAV's functional properties.

Characterization of radiolabeled capsid on viral proteins. The viral protein (VP) bands were visualized via protein staining (blue, 1st and 2nd lane), and the radiolabeled VP bands were imaged with sodium dodecyl sulfate-polyacrylamide gel electrophoresis (SDS-PAGE) and autoradiography (gray, 3rd lane) (Fig. 2e). The band location of the three VPs (blue bands) between Tz-PHP.eB and ${ }^{64} \mathrm{Cu}-\mathrm{PHP} . e B, \mathrm{Tz}-\mathrm{AAV} 9$ and ${ }^{64} \mathrm{Cu}-$ AAV9 and HS-AAV9-TC and ${ }^{64} \mathrm{Cu}-\mathrm{AAV} 9-\mathrm{TC}$ were similar. The relative radiolabeling of VP3 was greater than VP1 or VP2 for PHP.eB and AAV9 (Fig. 2e), directly related to the ratio of protein abundance for the three VPs, 1:1:10 (VP1, VP2, VP3). AAV9-TC was generated by site-specific insertion of the HRWCCPGCCKTF peptide motif at the VP1/VP2 interface at the $139^{\text {th }}$ amino acid $^{24}$. As a result, the gel image from autoradiography of ${ }^{64} \mathrm{Cu}-\mathrm{AAV} 9-\mathrm{TC}$ showed the VP2 band as the major radiolabeled VP whereas the protein staining (blue) of ${ }^{64} \mathrm{Cu}-\mathrm{AAV} 9-\mathrm{TC}$ was similar to the ratio of each VP (VP1:VP2: VP3, 1:1:10) (right column image of Fig. 2e).

PHP.eB size was unchanged after ${ }^{63} \mathrm{Cu}$-multichelator labeling The size of ${ }^{63} \mathrm{Cu}$-labeled and unlabeled PHP.eB was $27.9 \pm 0.64$ and $27.1 \pm 0.68 \mathrm{~nm}(n=3)$, respectively, and detected as a single peak (Supplementary Fig. 6).

Proteomic analysis of modified lysines on the capsid protein. We first determined the lysine sites modified with Tz-NHS by proteomic analysis. Mass lists from the analyses of excised gel bands of VP1, VP2, and VP3 after reaction of PHP.eB with TzNHS showed that tetrazines were predominantly incorporated on two lysines (K557 and K567) (Supplementary Table 2), which exist in all VPs. Importantly, the lysine at the 595th amino acid located within the engineered peptide sequences (DGTLAVPFK), critical for the distribution of PHP.eB to the brain and transduction of its transgene ${ }^{3}$, remained unreacted. Based on the crystal structure of AAV9 ${ }^{30}$, neither of the modified sites are located in the core of the threefold-proximal spikes, the region considered to be responsible for most virus-host interactions. $\mathrm{K} 567$ is located in the valley between the spikes, while $\mathrm{K} 557$ is on the distal shoulder (Fig. 2f). To the best of our knowledge, the two sites have not been reported to be involved in receptor binding for AAV9 derivatives.

Determination of the number of AAV labels. We compared results from optical labeling and electron microscopy to determine the number of labels. We examined the number of labels per 
a
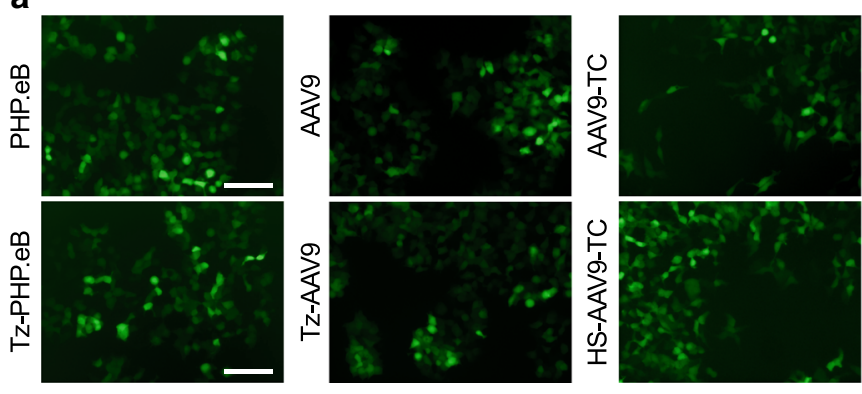

C

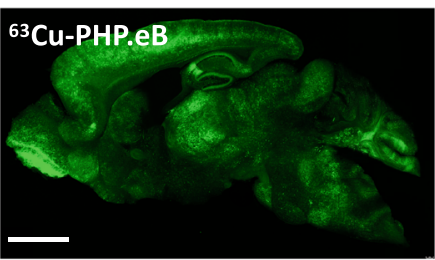

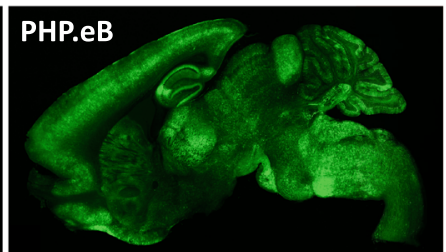

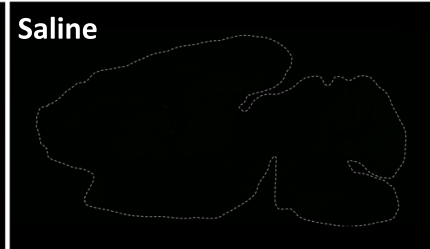

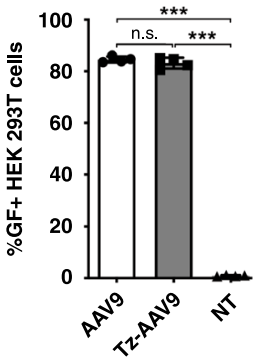
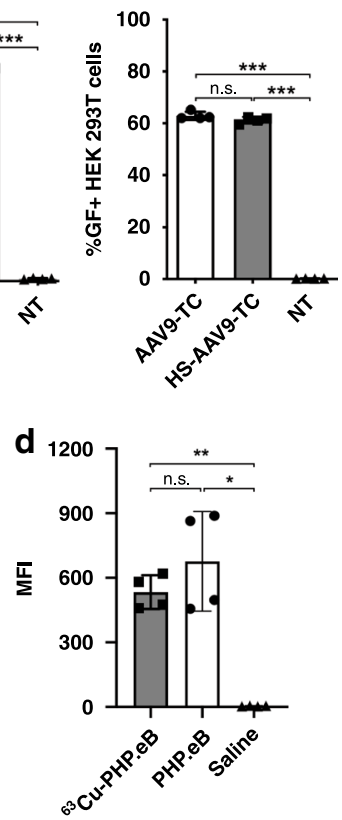

e
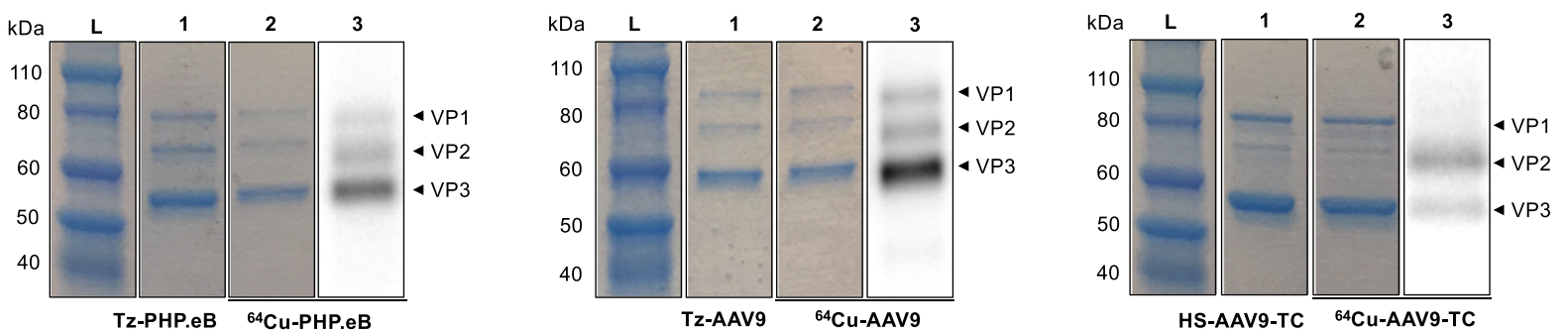

f
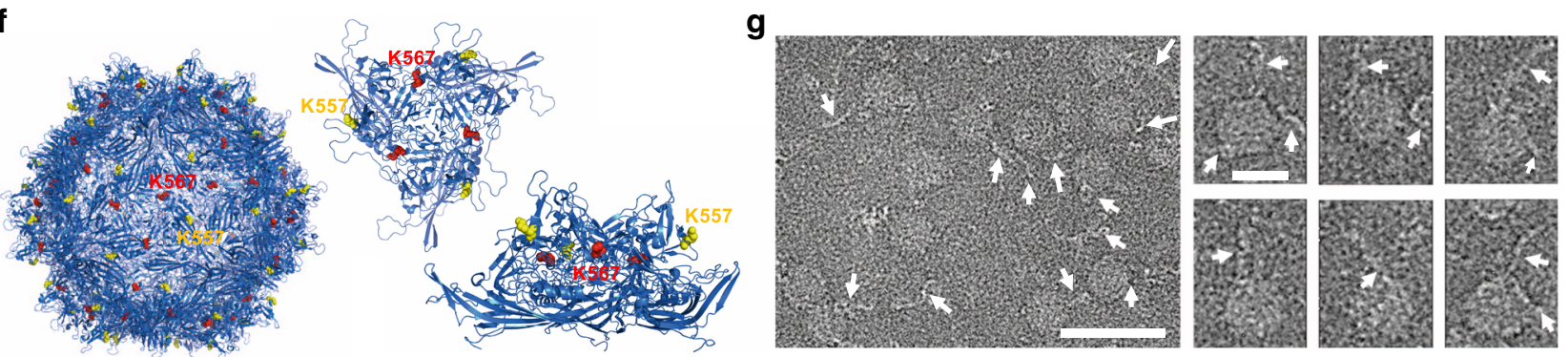

Fig. 2 Transduction and labeling efficiency of surface modified AAVs. a Fluorescence microscopy images of human embryonic kidney(HEK) 293T cells after $24 \mathrm{~h}$ incubation with intact AAVs (upper row, PHP.eB, AAV9, and AAV9-TC) and corresponding modified AAVs (lower row, Tz-PHP.eB, Tz-AAV9, and HS-AAV9-TC) at $1 \times 10^{6} \mathrm{AAV} /$ cell. b Percentage of green fluorescent positive $\left(\mathrm{GF}^{+}\right)$HEK293T cells 2 days after incubation with unmodified AAVs (PHP.eB, AAV9, and AAV9-TC, white bar with black circles) and the corresponding modified AAVs (gray bar with black squares), assessed by flow cytometry. The frequency of GF+ cells treated with unmodified and modified AAVs was similar and distinct from non-treated (NT, black triangles) cells ( $n=4$ per group). c Representative GFP images of sagittal brain sections from a C57BL/6 mouse at 3 weeks after tail vein administration of ${ }^{63} \mathrm{Cu}-\mathrm{PHP}$.eB, PHP.eB $\left(1.5 \times 10^{10} \mathrm{vg}\right)$ or saline (negative control) and $\mathbf{d}$ mean fluorescence intensity (MFI) of sagittal brain sections ( ${ }^{63} \mathrm{Cu}-\mathrm{PHP} . \mathrm{eB}$ : gray bar with black squares, PHP.eB: white bar with black circles, saline: black triangles, $n=4)$. e SDS-PAGE of modified AAVs (Tz-PHP.eB, Tz-AAV9, and HS-AAV9-TC; lane 1) and radiolabeled AAVs $\left({ }^{64} \mathrm{Cu}-\mathrm{PHP} . \mathrm{eB},{ }^{64} \mathrm{Cu}-\mathrm{AAV} 9\right.$, and ${ }^{64} \mathrm{Cu}-\mathrm{AAV} 9-\mathrm{TC}$; lane 2 and 3). The three bands depict viral protein (VP) 1-3 (L: standard protein ladder). Lane 1-3 illustrate blue-stained VPs (lanes 1 and 2) and radiolabeled VPs (lane 3), respectively. $\mathbf{f}$ Illustration of AAV9 capsid with modified lysines. Left: full view of AAV9, middle and right: top and side views of trimer viral proteins, respectively. The K557 (yellow) and K567 (red) lysine residues are highlighted. $\mathbf{g}$ Field view of direct-electron cryoEM images of PEG(40 kDa)-AAV9 (left image) and enhanced projection images of selected PEG(40 kDa)-AAV9 capsids (six right images). White arrows mark the $40 \mathrm{kDa}$ PEG molecules extended from the selected AAV capsids. Data are shown as mean \pm SD. Brown-Forsythe and Welch ANOVA with Dunnett's T3 multiple comparison test compares means (b, d). Significance is presented as n.s. (not significant), ${ }^{\star} P \leq 0.05,{ }^{\star \star} P \leq 0.01$, and ${ }^{\star \star \star} P \leq 0.001$. Whole gel and gel autoradiography images and $P$ values are shown in the source data. Scale bars: $100 \mu \mathrm{m}$ (a), $2 \mathrm{~mm}$ (c), $50 \mathrm{~nm}$ (g, left), $20 \mathrm{~nm}$ (g, right). 
capsid using the multi-armed fluorescent label (Supplementary Figs. $2 \mathrm{~b}$ and $7 \mathrm{a}$ ) combined with quantification of the AAV concentration through a titer. We applied this approach for both the amine- and thiol-directed coupling approaches. Labeling with 200-350 equivalents of Tz-NHS and reaction with 10-15 equivalents of (NOTA $)_{8}$-A555-TCO gave $5.4 \pm 2.3(n=6)$ and $3.5 \pm 3.0(n=8)$ labels per PHP.eB and AAV9 capsid, respectively (Supplementary Table 3 ). AAV9-TC reduced by 100 equivalents of TCEP and subsequently reacted with 20 equivalents of A555C2 maleimide yielded $0.5 \pm 0.3(n=4) \mathrm{ea} / \mathrm{vg}$ of A555-AAV9-TC (Supplementary Table 3).

Furthermore, since the $5 \mathrm{kDa}$ size of the (NOTA) ${ }_{8}$-A555 conjugate on AAV9 was not reliably visualized on cryogenic electron microscopy (cryoEM), we conjugated a larger label (PEG $(40 \mathrm{kDa})-\mathrm{TCO})$ (Supplementary Fig. 7b) to the capsid. This label was conjugated to AAV9 (denoted PEG(40 kDa)-AAV9) using the same conditions used for the in vivo imaging and was used to visualize the number of labels per virus (Supplementary Fig. 7a). PEG(40 kDa)-AAV9 was obtained from a reaction with Tz-AAV9 and 4 equivalents of PEG(40 kDa)-TCO and showed one to three labels per capsid on cryoEM images (Fig. 2g).

AAV radiolabeling was achieved with high radiochemical purity. The $20-35 \mathrm{pmol}$ of (NOTA) $)_{8}$-TCO and (NOTA $)_{8}-\mathrm{MI}$ were enough to incorporate $>99 \%$ of $1-2 \mathrm{mCi} \mathrm{Cu}-64$. In situ incubation of Tz-AAV9 and -PHP.eB and HS-AAV9-TC with these multichelators yielded ${ }^{64} \mathrm{Cu}-\mathrm{AAV} 9,-\mathrm{AAV} 9-\mathrm{TC}$, and -PHP. eB to $2.2 \%(1.78 \mathrm{MBq}(48 \mu \mathrm{Ci})), 6.6 \%(4.88 \mathrm{MBq}(132 \mu \mathrm{Ci})$ and $7.5 \pm 6 \%(3.9 \pm 1.7 \mathrm{MBq}(106 \pm 45 \mu \mathrm{Ci}))$, respectively (decay corrected). Radiochemical purity of radiolabeled AAVs on instant radio-thin layer chromatography was above $98 \%$.

PET imaging quantified brain accumulation and receptor binding. The PK and biodistribution of the ${ }^{64} \mathrm{Cu}-\mathrm{AAV} 9,-\mathrm{AAV} 9-$ TC, and -PHP.eB capsids (as defined in Fig. 1) were assessed in C57BL/6 mice ( $n=3$ /group) with PET/CT as illustrated in Fig. 3a. The projection images acquired of AAVs revealed two remarkable distinctions: the high brain uptake of PHP.eB and the extended blood circulation of AAV9 (Fig. 3b, Supplementary Movies 1-3). Blood circulation of $\operatorname{AAV} 9\left(t_{1 / 2}=5.0 \mathrm{~h}\right)$ was longer than that of AAV9-TC $\left(t_{1 / 2}=2.4 \mathrm{~h}\right)$ and PHP.eB $\left(t_{1 / 2}=3.1 \mathrm{~h}\right)$ (Fig. 3c, Supplementary Tables 4 and 5). The faster clearance of PHP.eB from blood, as compared to AAV9, is expected due to rapid uptake within the brain (Supplementary Movie 2a at $4 \mathrm{~h}$ ). The mechanism for the enhanced clearance of AAV9-TC has not been fully characterized; however, the tetracysteine motif (HRWCCPGCCKTF) on AAV9-TC can remain reactive after reduction by TCEP, and S-thiolation by serum proteins ${ }^{38}$ can reduce stability, potentially resulting in a protein corona or aggregation over time $\mathrm{s}^{39}$. Thus, while the initial $(30 \mathrm{~min})$ receptor binding is expected to be similar, clearance from blood through the liver and intestine (over hours) is expected to be enhanced for AAV9-TC (Supplementary Movie 3a at $4 \mathrm{~h}$ ). Brain accumulation of PHP.eB was greater at all time points than that of AAV $(n=$ $3, P=0.0096$ at $0 \mathrm{~h}, P=0.0004$ at $4 \mathrm{~h}, P=0.0007$ at $21 \mathrm{~h})$ and AAV9-TC $(n=3, P=0.0116$ at $0 \mathrm{~h}, P=0.0003$ at $4 \mathrm{~h}, P=0.0006$ at $21 \mathrm{~h}$ ) (Fig. 3c, Supplementary Table 6). Maximum uptake (\% $\mathrm{ID} / \mathrm{cc}$ ) of ${ }^{64} \mathrm{Cu}-\mathrm{PHP} . \mathrm{BB}$ in the entire brain was $35 \% \mathrm{ID} / \mathrm{cc}$, with the spatial maximum observed in the midbrain (Fig. 3d) and strong midbrain uptake clearly visualized in the projected PET/ CT brain image (Fig. 3e). In addition, in order to assess the PK of multichelator-labeled PHP.eB compared to that of PHP.eB, we performed classical qPCR. Un-labeled PHP.eB and (NOTA) $8^{-}$ A555-PHP.eB cleared at a similar rate from the blood pool over $21 \mathrm{~h}\left(t_{1 / 2}=4.8 \mathrm{~h}\right.$ vs $5.3 \mathrm{~h}$, respectively) (Fig. $3 \mathrm{f}$, left,
Supplementary Tables 4 and 7) and the blood clearance was similar to that observed by PET (Fig. 3c). The biodistribution of PHP.eB and (NOTA) $)_{8}$-A555-PHP.eB was similar in the major organs such as the brain, heart, liver, spleen, kidney and blood (Fig. 3f, right, Supplementary Table 8).

Sliced sagittal brain images in the cerebral cortex, thalamus, midbrain and cerebellum from PET/CT (after $21 \mathrm{~h}$ ), autoradiography (after $21 \mathrm{~h}$ ), and ex vivo GFP fluorescence (after 3 weeks) showed a consistent distribution of the viral capsid tag and corresponding transduced GFP protein expression (Fig. 3g, Supplementary Fig. 8). PHP.eB accumulation in the brain was then analyzed in dynamic 5-min intervals over the first $30 \mathrm{~min}$. $4 \%$ and $10 \% \mathrm{ID} / \mathrm{cc}$ of PHP.eB was bound at 5 and $30 \mathrm{~min}$, respectively, whereas AAV9 and AAV9-TC accumulation was $<1 \%$ ID/cc (Fig. 3h, Supplementary Fig. 8). Furthermore, Logan plots, based on a reversible accumulation model, demonstrated that the $30 \mathrm{~min}$ accumulation in brain was greater for PHP. eB»AAV9 AAV9-TC, with a distribution volume of 0.210, 0.011 , and 0.011 , respectively (Fig. $3 \mathrm{~h}$ ). Thus, the affinity of PHP. $\mathrm{eB}$ for the brain endothelium is estimated to be 20-fold higher than for AAV9 and AAV9-TC. The receptor affinity of AAV9 was identical for the two labeling methods as assessed by the initial 30-min Logan plot (Fig. 3h).

Multichelator does not alter PHP.eB endothelial accumulation. We employed two optical probes: a probe in which an optical dye (A555-NHS ester) was attached to the native capsid lysines and a second optical probe $\left((\mathrm{NOTA})_{8}-\mathrm{A} 555-\mathrm{TCO}\right)$ conjugated to the multichelator construct in a manner similar to the (NOTA) $8^{-}$ TCO conjugate (Supplementary Fig. 7a). The binding of A555PHP.eB, A555-AAV9 (Supplementary Fig. 9), (NOTA) 8 -A555PHP.eB (Fig. 3i) or (NOTA) ${ }_{8}$-A555-AAV9 (Supplementary Fig. 10) to the brain endothelium, observed by confocal microscopy at 4, 24, and $48 \mathrm{~h}$ after injection (Z-stack images in Supplementary Movie 4), showed that punctate clusters were observed at $4 \mathrm{~h}$ after injection. The fluorescence intensity gradually diminished by $24 \mathrm{~h}$ and was similar to saline injection (Supplementary Fig. 10) at 48 h. Taken together, the optical and PET images suggest that the early-bound PHP.eB crossed the BBB within $48 \mathrm{~h}$ and specific and effective binding of PHP.eB to the brain endothelium was confirmed.

PET imaging elucidates strain and treatment-dependent PK. Since mouse strain dependence of PHP.eB BBB transcytosis has been reported ${ }^{10,40}$, the $\mathrm{PK}$, brain uptake and biodistribution of ${ }^{64} \mathrm{Cu}-\mathrm{PHP} . \mathrm{eB}$ were assessed by comparing BALB/c and C57BL/6 mice (Fig. 4a, Supplementary Table 9). Dramatically-reduced brain uptake of ${ }^{64} \mathrm{Cu}$-PHP.eB was confirmed in BALB/c with respect to $\mathrm{C} 57 \mathrm{BL} / 6$ mice from the time-activity curve over $21 \mathrm{~h}$ (Fig. 4a) $(n=3, P=0.0048$ at $0 \mathrm{~h}, P=0.0002$ at $4 \mathrm{~h}, P=0.004$ at $21 \mathrm{~h}$ ) and PET/CT images at $0 \mathrm{~h}$ (Fig. $4 \mathrm{~b})$. Similar results (\% ID/g) were observed in the brain radioactivity from the biodistribution at $21 \mathrm{~h}$ (Fig. $4 \mathrm{c}, n=3, P=0.0193$ ). In addition, the uptake of PHP.eB was greater in the liver of $\mathrm{BALB} / \mathrm{c}$ than $\mathrm{C} 57 \mathrm{BL} / 6$ mice $(n=3, \quad P=0.0627$ at $4 \mathrm{~h})$ (Fig. $4 \mathrm{~d}$, e and Supplementary Table 10). While the enhanced liver accumulation is anticipated given the lack of brain accumulation, a strain-specific immune response has also been reported to enhance liver accumulation in the BALB/c strain ${ }^{41,42}$. Further, the circulation time of PHP.eB $(3.1 \mathrm{~h})$ in the BALB/c mouse $(2.4 \mathrm{~h})$ was slightly lower than that in C57BL/6 mice (Fig. 4a, Supplementary Table 4). The results reaffirm the reduced brain uptake in BALB/c mice, which, unlike C57BL/6 mice, lack the LY6A receptor that the engineered PHP. $\mathrm{eB}$ binds to ${ }^{10}$; however, other differences also exist in the $\mathrm{PK}$ between strains. 
a

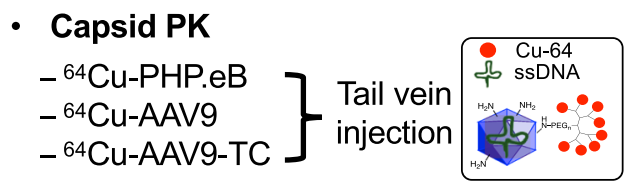

$\mathrm{PET} / \mathrm{CT}$ O $\mathrm{h} \quad 4 \mathrm{~h} \longrightarrow$

$21 \mathrm{~h} \cdot$ biodistribution b ${ }^{64}$ Cu-AAV9 $\quad{ }^{64} \mathrm{Cu}$-AAV9-TC ${ }^{64}$ Cu-PHP.eB $\quad{ }^{64} \mathrm{Cu}-\mathrm{AAV} 9 \quad{ }^{64} \mathrm{Cu}-\mathrm{AAV} 9-\mathrm{TC} \quad{ }^{64} \mathrm{Cu}-\mathrm{PHP} . \mathrm{BB}$

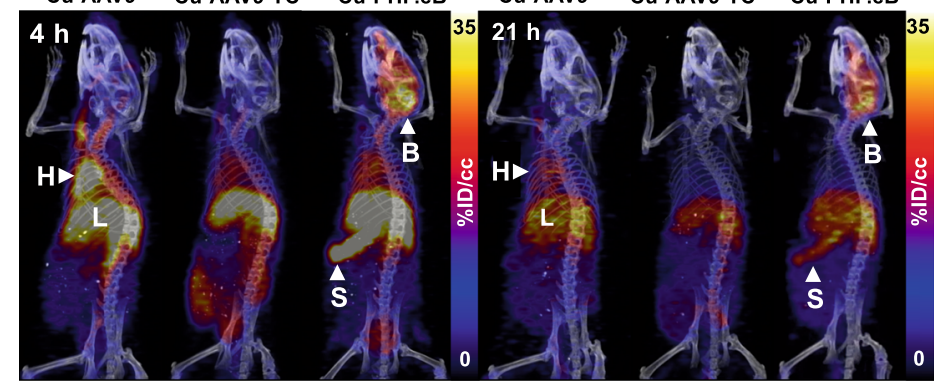

C

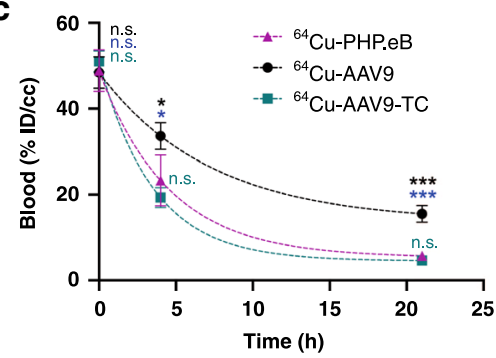

f

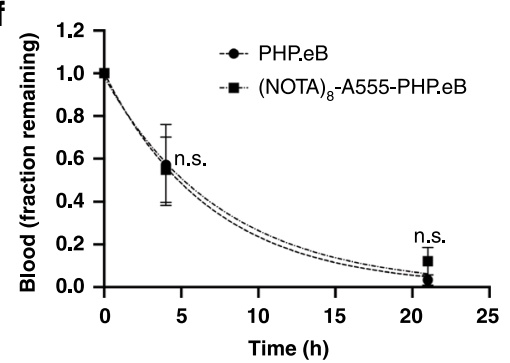

g

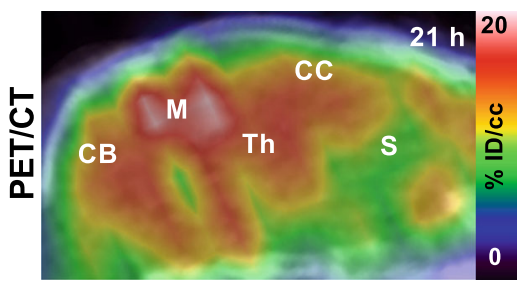

h

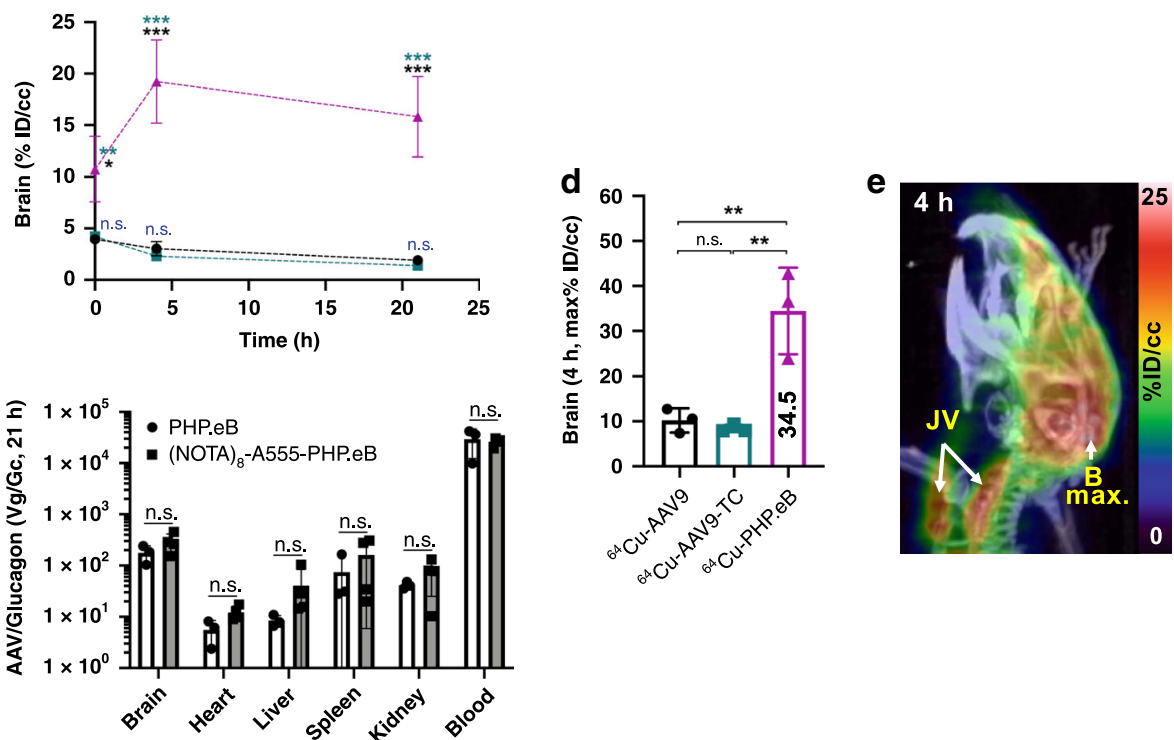

Previously, pretreatment with neuraminidase (NA) in vitro and in vivo, was reported to expose terminal $\mathrm{N}$-linked galactose and enhance AAV9 binding ${ }^{43,44}$. In our study, nasal administration of $\mathrm{NA}$ was followed by IV injection of ${ }^{64} \mathrm{Cu}-\mathrm{PHP} . \mathrm{eB}$ and biodistribution at $21 \mathrm{~h}$ in $\mathrm{BALB} / \mathrm{c}$ mice. Lung and brain accumulation were increased by NA administration $1.4(n=3$, $P=0.0229)$ and 2.0 -fold $(n=3, P=0.0364)$, respectively (Fig. 4f). The treatment by NA also increased the PHP.eB circulation time from $2.4 \mathrm{~h}$ to $5.6 \mathrm{~h}$ (Fig. $4 \mathrm{a}$ ). However, the increased accumulation was relatively small as compared to the differences in brain accumulation of PHP.eB between strains.

\section{Discussion}

We found that radiolabeling AAVs with a unique multichelator construct allows for a detailed and quantitative study of AAV biodistribution and pharmacokinetics. Following conjugation of a dendrimer-like radioactive tag to novel AAVs, the fraction of 
Fig. 3 PET and optical imaging-based assessment of AAV pharmacokinetics in C57BL/6 mice. a Experimental setup for region of interest (ROI) analysis $(0,4$, and $21 \mathrm{~h})$ and biodistribution $(21 \mathrm{~h})$ of ${ }^{64} \mathrm{Cu}-\mathrm{PHP} . \mathrm{eB},-\mathrm{AAV} 9$, and -AAV9-TC. PET images are acquired at 0,4 and $21 \mathrm{~h}$ after AAV tail vein administration. b Projected PET/CT images at 4 (left) and $21 \mathrm{~h}$ (right) ( $\mathrm{H}$ heart, L liver, S spleen, B brain). c Time activity curves (over $21 \mathrm{~h}$ ) and $\mathbf{d}$ maximum brain uptake (at $4 \mathrm{~h}$ ) of ${ }^{64} \mathrm{Cu}-\mathrm{PHP} . \mathrm{eB}$ (magenta triangle), ${ }^{64} \mathrm{Cu}$-AAV9 (black circle), and ${ }^{64} \mathrm{Cu}-\mathrm{AAV} 9-\mathrm{TC}$ (turquoise square) from the ROI analysis of blood and brain $(n=3)$ after tail vein administration. e Representative projected PET/CT image at $4 \mathrm{~h}$ of ${ }^{64} \mathrm{Cu}-\mathrm{PHP}$.eB within the brain (B brain, JV jugular vein). f PK (left) and 21-h biodistribution (right) of PHP.eB $(n=3 \text {, black circle) and (NOTA) })_{8}$-A555-PHP.eB ( $n=4$, black squares) obtained by qPCR. $\mathbf{g}$ Sliced $\mathrm{PET} / \mathrm{CT}$, autoradiography and GFP images of sagittal section of mouse brain (CB cerebellum, M midbrain, Th thalamus, CC cerebral cortex, $\mathrm{S}$ striatum) acquired at $21 \mathrm{~h}, 21 \mathrm{~h}$ and 3 weeks, respectively, after tail vein injection of ${ }^{64} \mathrm{Cu}-\mathrm{PHP} . \mathrm{eB}$ for PET/CT and autoradiography and non-radioactive ${ }^{63} \mathrm{Cu}-\mathrm{PHP} . \mathrm{eB}$ for the GFP image. $\mathbf{h}{ }^{64} \mathrm{Cu}-\mathrm{AAVs}$ brain accumulation ( $n=3$ per group) measured 30 min after tail vein administration (left) and Logan plots (right) of brain uptake rate after AAV administration. i Representative confocal images of (NOTA) 8 -A555-PHP.eB (red) on brain endothelium (green) acquired 4, 24, and $48 \mathrm{~h}$ after tail vein injection. White arrows indicate (NOTA) 8 -A555-PHP.eBs (red). Data are shown as mean \pm SD. One-way ANOVA with Tukey's multiple comparison test (c, $\mathbf{d}$, and $\mathbf{h}$ (left)) compared means of the three groups. Multiple unpaired $t$-tests with the Holm-Sidak method with alpha $=0.05$ compared the means in $\mathbf{f}$. Significance: n.s. (not significant), ${ }^{\star} P \leq 0.05,{ }^{\star \star} P \leq 0.01$, and ${ }^{\star \star \star} P \leq 0.001$. $P$ values are shown in the source data. Intensity values in $\mathbf{b}, \mathbf{d}, \mathbf{e}$, and (g, left) are percent injected dose per cubic centimeter (\% ID/cc). Scale bars: $2 \mathrm{~mm}(\mathbf{g}), 25 \mu \mathrm{m}(\mathbf{i})$.

a

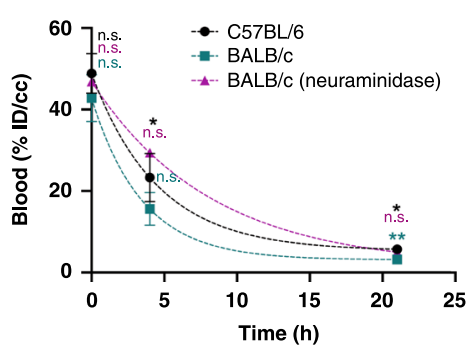



b

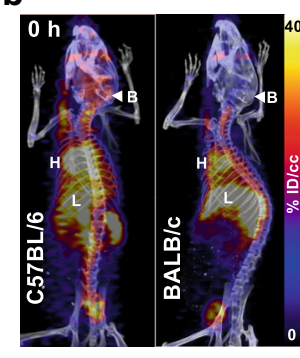

C
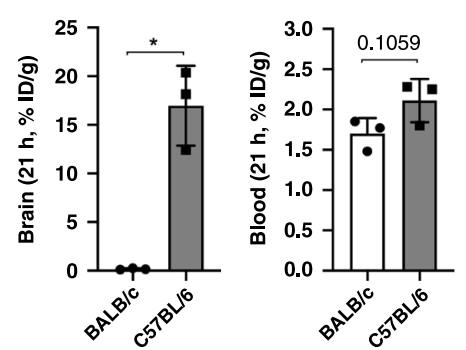


Fig. 4 Strain and neuraminidase-dependent pharmacokinetics of ${ }^{64}$ Cu-PHP.eB. a Time activity curves of PHP.eB obtained from region of interest (ROI) analysis of blood (left) and brain (right) from C57BL/6 (black circle), BALB/c (turquoise square), and neuraminidase-treated BALB/c (magenta triangle) mice over $21 \mathrm{~h}$ ( $n=3$ per group). Radioactivity from ROI analysis is presented as \% ID/cc. b Representative PET/CT projection images (B brain, $\mathrm{H}$ heart, $\mathrm{L}$ liver) acquired over $30 \mathrm{~min}$ after tail vein injection of ${ }^{64} \mathrm{Cu}-\mathrm{PHP} . \mathrm{eB}$ to $\mathrm{C} 57 \mathrm{BL} / 6$ and BALB/c mice. c Biodistribution (\% ID/g) of ${ }^{64} \mathrm{Cu}-\mathrm{PHP} . \mathrm{eB}$ in brain (left) and blood (right) in C57BL/6 (gray bar with black squares) and BALB/c (white bar with black circle) mice at $21 \mathrm{~h}(n=3)$. d Sliced PET/CT image (H heart, $\mathrm{L}$ liver) at $4 \mathrm{~h}$ after tail vein injection of ${ }^{64} \mathrm{Cu}-\mathrm{PHP} . \mathrm{eB}$. e Time activity curve of ${ }^{64} \mathrm{Cu}-\mathrm{PHP} . \mathrm{eB}$ measured from C57BL/6 (black circle) and BALB/c (turquoise square) livers $(n=3)$. f Biodistribution ( $21 \mathrm{~h})$ of ${ }^{64} \mathrm{Cu}-\mathrm{PHP} . \mathrm{eB}$ in blood (left), brain (middle), and lung (right) from BALB/c with no treatment white bar with black circle) versus BALB/c mice treated with neuraminidase (gray bar with black square) $(n=3)$. Data are shown as mean \pm SD. For statistical analysis, a one-way ANOVA with Tukey's multiple comparison test in a was performed to compare means of three groups (C57BL/6 vs BALB/c: turquoise, C57BL/6 vs BALB/c (neuraminidase): magenta, BALB/c vs BALB/c (neuraminidase): black) at each time point. Unpaired two-tailed Welch's $t$-test was performed in c, $(\mathbf{e}, 0,4$, and $21 \mathrm{~h})$ and $\mathbf{f}$. Significance is presented as ${ }^{\star} P \leq 0.05,{ }^{\star}{ }^{\star} P \leq 0.01$. $P$ values are shown in the source data. Maximum and minimum intensity values of $P E T / C T$ images in $\mathbf{b}, \mathbf{d}$ are presented as percent injected dose per cubic centimeter (\% ID/cC).

radioactivity delivered to the brain was extraordinarily high, thus demonstrating the potential use of AAVs as carriers. PET, with appropriate analytic methods, was applied to noninvasively quantify the endothelial receptor binding with a resolution of minutes. We found that Logan analysis is particularly important for characterizing the binding of novel AAV capsids as a rapid and largely irreversible accumulation occurs on the endothelium of the target tissue. No alternative technique can quantify binding of viral capsids in real time; such information is critically important for capsid engineering and for the identification of the endothelial receptors responsible for AAV accumulation and transcytosis.
Recent reports indicate that enhanced brain transduction by PHP.eB in C57BL/6 mice requires an LY6A receptor-mediated pathway that is independent of galactose and is absent in $\mathrm{BALB} / \mathrm{c}$ mice and non-human primates ${ }^{10} .{ }^{64} \mathrm{Cu}-\mathrm{PHP} . \mathrm{eB}$ can be exploited as a non-invasive tool to measure endothelium binding mediated by the LY6A receptor in various mouse strains and other species. Furthermore, the binding of serotype AAVs to the AAV receptor (AAVR), identified as a critical host factor for infection of naturally-occurring $\mathrm{AAVs}^{45}$, can be assessed by a simple blocking study with novel AAVs.

Optical imaging of the tagged capsid validated the accumulation of the capsids on the brain endothelium within minutes and 
the elimination of the punctate fluorescence on the endothelium over $24 \mathrm{~h}$. Given the very small size of the AAVs $(25 \mathrm{~nm})$, tracking of their fluorescence within the brain was not feasible. PET imaging complemented the information in the optical image by demonstrating that the accumulated radioactivity remains within the brain over $21 \mathrm{~h}$. Classical pharmacokinetic analysis based on a PCR further validated the brain accumulation. Given that the radioactivity was tagged to the capsid surface, the distribution of radioactivity throughout the brain suggests that the capsids were transcytosed across the BBB. This observation supports previous reports of AAV transcytosis across the BBB, as observed in vitro ${ }^{46}$. Combined PET and optical reporter gene imaging demonstrated that the pattern of transduction within the brain was similar to the distribution of the radioactive tag.

The stoichiometry of fluorophore-TCO conjugates on the surface of AAV9 and PHP.eB was on average four labels per particle. Labeling of a fluorescence-maleimide conjugate on AAV9-TC conferred 0.6 label per capsid. CryoEM of AAV9 conjugated with PEG $(40 \mathrm{kDa})$ similarly showed $2-3$ copies of extended PEG-string density per capsid. Our AAV surface modification with tetrazine-NHS ester retained transduction efficiency in HEK cells and in in vivo transduction studies. Following conjugation with the $5 \mathrm{kDa}$ multichelator at a multichelator:AAV ratio of 1:2 PHP.eB, binding to the brain endothelium and transduction were maintained. Future work will focus on optimizing the number and size of the conjugated tags. Here, we minimized the number of tags per AAV in the in vivo studies of transduction to minimize any effect on transport. Given the relatively small loading capacity of AAVs, the potential to conjugate additional cargo to the capsid could be transformative. Multiple gene editing components, complementary therapeutics or additional imaging tags can be attached to the surface.

The predominantly-labeled lysines of each viral protein within PHP.eB were K557 and K567. K61, K92, K528, K618, K696, and K700 were modified in a smaller fraction of capsids (Supplementary Table 2). We speculate that K557 and K567 are susceptible to reaction with the NHS-ester and that this is the basis of their enhanced modifications. The variable region VII (aa545aa558), including K557, is located within a region of the AAV capsid associated with liver transduction ${ }^{47}$ and delayed blood clearance $^{30,33}$; however, to our best knowledge, there is no report on the direct involvement of $\mathrm{K} 557$ or K567 in host receptor binding. Recently, unnatural amino acids (UAA) bearing an azide were site-specifically engineered in AAV2 (at aa 587) and AAVDJ (a derivative of AAV8, at aa 589) capsids and utilized to conjugate oligonucleotides ${ }^{48}$. Specific protocols can also be developed for labeling of other capsids. For example, there is a lysine adjacent to K557 on AAV2 and adjacent to K567 on AAV1, $6,8,9$, and 10 (Supplementary Table 2). Therefore, addition or substitution of lysines, cysteines or UAAs within these sequences or other AAVs can also provide a unique labeling site.

Finally, the PET method developed here to monitor binding and pharmacokinetics will be paired with PET reporter gene imaging in future work. A PET reporter gene based on pyruvate kinase (PKM2) has been shown to have low background in the brain and can be packaged within AAVs ${ }^{49}$. PKM2 can be used with the reporter probe $\left[{ }^{18} \mathrm{~F}\right] \mathrm{DASA}-23^{49}$, which is permeable to the blood-brain barrier in order to monitor brain transduction over months or years. In the future, we will couple this tracer with the PET tag described here.

\section{Methods}

Materials and reagents. All solvents were purchased from Fisher Chemical, Sigma-Aldrich and Acros. The reagents and materials for multichelator synthesis were purchased from Novabiochem, Click Chemistry Tools and Biotage. PEG 27 spacers were purchased from Chem-Impex International Inc. and Polypure. PEG
(40 kDa)-amine (Creative PEGWorks) was purchased from Fisher Scientific. For the capsid SDS-PAGE, the gels, buffer, standard ladder and protein staining reagents were purchased from ThermoFisher Scientific. AFDye555-maleimide (Fluoroprobes), AlexaFluor555-NHS ester (ThermoFisher Scientific) and AlexaFluor555-C2-maleimide (ThermoFisher Scientific), each with 555 excitation max and 580 emission max, are denoted as A555 throughout. The detailed list of materials and reagents is in the Supplementary Methods.

Cell line and AAVs. Human embryonic kidney cells (HEK293T) were obtained from ATCC (CRL-1573). AAV9, AAV9-TC, and PHP.eB packaging including CAG-mNeonGreen or CAG-DIO-GFP were prepared as described in the Supplementary Methods section entitled "Preparation of AAV9, AAV9-TC, and PHP.eB". All AAVs were used within two months of preparation. AAV-PHP.eB packaging CAG-GFP was purchased from Addgene (\#37825-PHP.eB). All AAVs used for in vitro/in vivo transduction, PET/CT, and optical studies are summarized in Supplementary Table 11.

Synthesis of (NOTA) $)_{\mathbf{8}}-\mathbf{N H}_{\mathbf{2}}$. Branched $\left(\mathrm{NH}_{2}\right)_{8}-\mathrm{NH}_{2}$ was synthesized on rink amide resins $(0.49 \mathrm{mmol} / \mathrm{g}, 0.15 \mathrm{~g})$ in a microwave-assisted solid phase synthesizer (Initiator + Alstra, Biotage) as shown in Supplementary Fig. 2a. Sequential coupling reaction was programed to be performed at $75^{\circ} \mathrm{C}$ for 5 min with Fmoc-lys (Boc)- $\mathrm{OH}$ ( 3 equivalents, $1.47 \mathrm{mmol}, 113 \mathrm{mg}$ ), $0.2 \mathrm{M}$ Fmoc- $\mathrm{PEG}_{27}-\mathrm{OH}$ (3 equivalents, $1.47 \mathrm{mmol}, 372 \mathrm{mg}$ ), $0.2 \mathrm{M}$ Fmoc-lys(Fmoc)-OH (3 equiv., $1.47 \mathrm{mmol}$ $131 \mathrm{mg}$ ), 0.2 M Fmoc-lys(Fmoc)-OH (5 equivalents, $2.45 \mathrm{mmol}, 219 \mathrm{mg}$ ), and 0.2 M Fmoc-lys(Fmoc)-OH (4.9 equivalents, $439 \mathrm{mg}$ ) with 0.1 or $0.5 \mathrm{M} \mathrm{HBTU}$ (one equivalent of each amino acids) and $0.2 \mathrm{M}$ DIPEA (two equivalents of each amino acids). The volume of each coupling reaction was maintained to be 3-5 mL DMF. After drying resins under a vacuum, NOTA-bis(t-bu ester) (10 equivalents of primary amine on resin, $100 \mathrm{mg}, 0.24 \mathrm{mmol}$ ) was manually further coupled to the lysine residue (eight amines per mole loading level, $0.25 \mathrm{mmol} / \mathrm{g}, 100 \mathrm{mg}, 0.025$ $\mathrm{mmol})$ on resin with HBTU $(89 \mathrm{mg}, 0.24 \mathrm{mmol})$ and DIPEA $(83 \mathrm{mg}, 0.64 \mathrm{mmol})$ in DMF $(2 \mathrm{~mL})$. NOTA-bis $(t$-bu ester) conjugation was monitored by TNBS assay. When the TNBS test was positive, NOTA-bis( $t$-bu ester) conjugation was performed one more time. After the cleavage of the (NOTA) $)_{8}-\mathrm{NH}_{2}$ mixture from resin in a cocktail of TFA (95\%), water (2.5\%), and TIPS (2.5\%), (NOTA) $)_{8}-\mathrm{NH}_{2}(n=2$, $13 \pm 2 \mathrm{mg}, 2.8 \mu \mathrm{mol}$ ) was isolated by HPLC (acetonitrile gradient from $5 \%$ to $60 \%$ with $0.1 \% \mathrm{TFA}$ solution for $30 \mathrm{~min}$, retention time: $15.5 \mathrm{~min}$ ). The mass of (NOTA) $)_{8}-\mathrm{NH}_{2}$ was confirmed by MALDI-TOF $\left(\left[\mathrm{M}+\mathrm{H}^{+}\right]\right.$, exact mass was calculated at 4627.62 and found at 4627.20 Da) (Supplementary Fig. 2a).

Synthesis of (NOTA) $)_{8}$-transcyclooctene (TCO). To a solution of (NOTA) $)_{8}-\mathrm{NH}_{2}$ $(1,3.3 \mathrm{mg}, 0.7 \mu \mathrm{mol})$ in $1 \mathrm{x}$ PBS $(1 \mathrm{~mL}, \mathrm{pH} 7.8)$, transcyclooctene-PEG $\mathrm{P}_{4}-\mathrm{NHS}$ (TCO-PEG -NHS $10 \mathrm{mg}, 19 \mu \mathrm{mol}$,) dissolved in DMSO $(80 \mu \mathrm{L})$ was added. $\mathrm{pH}$ was readjusted to 8 , and the reaction mixture was stirred in a vortex mixer at room temperature for $2-3 \mathrm{~h}$. The solution was diluted with double distilled-water (3-4 mL) and concentrated using a $3 \mathrm{kDa}$ MWCO spin filter unit. Dilution and concentration steps were repeated. (NOTA) $)_{8}$ TCO $(2,1 \mathrm{mg}, 0.4 \mu \mathrm{mol})$ was isolated by HPLC (acetonitrile gradient from $5 \%$ to $60 \%$ with $0.1 \%$ TFA solution for $30 \mathrm{~min}$, retention time: $24.4 \mathrm{~min}$ ). Mass of (NOTA) ${ }_{8}$-TCO was confirmed by MALDI-TOF (Supplementary Fig. 2a).

Synthesis of (NOTA) $)_{\mathbf{8}}$-A555-TCO. (NOTA) $)_{8}$-cys(SH)- $\mathrm{NH}_{2}$ (4) was similarly synthesized by adding cysteine and a mono-PEG sequence between $\mathrm{PEG}_{27}$ and the lysine from (NOTA) $)_{8}$ TCO as shown in Supplementary Fig. 2b. After isolation of the product with HPLC, the MALDI-TOF spectrum confirmed the mass of $(\mathrm{NOTA})_{8}$-cys $(\mathrm{SH})-\mathrm{NH}_{2}$. (NOTA) ${ }_{8}$-cys $(\mathrm{SH})-\mathrm{NH}_{2}(2 \mathrm{mg}, 0.41 \mu \mathrm{mol})$ was reacted with AF555-maleimide ( $1 \mathrm{mg}, 0.79 \mu \mathrm{mol}$, Fluoroprobe, Az) in PBS, then the isolation with $3 \mathrm{kDa}$ MWCO spin filter and HPLC afforded (NOTA) $)_{8}-\mathrm{A}_{555}-\mathrm{NH}_{2}(5$, $1 \mathrm{mg}, 0.17 \mu \mathrm{mol})$. (NOTA) ${ }_{8}$-A555- $\mathrm{NH}_{2}(1 \mathrm{mg}, 0.17 \mu \mathrm{mol})$ reacted with TCO-PEG ${ }_{4}$ NHS $(3 \mathrm{mg}, 5.8 \mu \mathrm{mol})$ in $1 x P B S(1 \mathrm{~mL}, \mathrm{pH} 8)$ gave (NOTA) $)_{8}$ A555-TCO $(6,350$ $\mu \mathrm{g}, 0.06 \mu \mathrm{mol}$ ) after HPLC purification (Supplementary Fig. 2b). The AF555, the fluorophore in AF555-MI, is denoted as A555 in the conjugated form.

Synthesis of (NOTA) $)_{\mathbf{8}}$-maleimide (MI). To a solution of (NOTA) $)_{8}-\mathrm{NH}_{2}(1,2 \mathrm{mg}$ $0.7 \mu \mathrm{mol})$ in $1 \mathrm{x}$ PBS $(0.5 \mathrm{~mL}, \mathrm{pH} 7.4), 0.1 \mathrm{M}$ EDTA $(10 \mu \mathrm{L})$ and SM(PEG) 2 (NHS $\mathrm{PEG}_{2}$-MI, $\left.4 \mathrm{mg}, 9.4 \mu \mathrm{mol}\right)$ dissolved in DMSO $(30 \mu \mathrm{L})$ were added. The reaction mixture was stirred in a vortex mixer at room temperature for $1 \mathrm{~h}$. The solution was then diluted with $0.05 \%$ TFA in water $(3-4 \mathrm{~mL})$ and concentrated using a 3 $\mathrm{kDa}$ MWCO spin filter unit. Dilution and concentration steps were repeated. (NOTA) $)_{8}$-MI $(3,1 \mathrm{mg}, 0.2 \mu \mathrm{mol})$ was isolated by HPLC (acetonitrile gradient from $5 \%$ to $60 \%$ with $0.1 \%$ TFA solution for $30 \mathrm{~min}$, retention time: $16.6 \mathrm{~min}$ ). Mass of (NOTA $)_{8}$-MI) was confirmed by MALDI-TOF $\left(\left[\mathrm{M}+\mathrm{H}^{+}\right]\right.$, exact mass was calculated at 4937.74 and found at 4937.66) (Supplementary Fig. 2a).

Titration and LC-MS/MS. Detailed methods for the titration of single- and multichelators with Cu-63/Cu-64 (shown in Supplementary Fig. 3) and for LC-MS/ MS analysis of the Tz-PHP.eB capsid (shown in Supplementary Table 2) are available in the Supplementary Methods section. 
In vitro evaluation of PHP.eB transduction before and after surface amine modification with Tz-NHS ester. To PHP.eB:CAG-GFP $\left(6.3 \times 10^{12} \mathrm{vg}, 10 \mathrm{pmol}\right)$ in $1 \mathrm{x}$ PBS $\left(350 \mu \mathrm{L}, \mathrm{pH}\right.$ adjusted to 8 with $0.1 \mathrm{M} \mathrm{Na}_{2} \mathrm{CO}_{3}$ solution ( $\left.\left.\mathrm{pH} 9.1\right)\right), 5 \mathrm{mM}$ tetrazine- $\mathrm{PEG}_{5}$-NHS $(1 \mu \mathrm{L}, 5 \mathrm{nmol})$ in anhydrous DMSO was added. After incubation for $1.5 \mathrm{~h}$ at room temperature, the reaction mixture was dialyzed in a minidialysis device (20 kDa molecular weight cut-off (MWCO)) in $500 \mathrm{~mL} \mathrm{1x}$ PBS overnight at $4{ }^{\circ} \mathrm{C}$. The transduction of CAG-GPF by Tz-PHP.eB and PHP.eB was compared in HEK293T cells seeded at a density of $70 \%$ in a 24 -well plate $\left(3 \times 10^{4}\right.$ cells/well) with $500 \mu \mathrm{L}$ of Dulbecco's Modified Eagle's medium (DMEM; Invitrogen) supplemented with $10 \%$ fetal bovine serum (FBS; Invitrogen) and $1 \%$ penicillin-streptomycin (Invitrogen) (DMEMc). After incubation at $37^{\circ} \mathrm{C}$ and $5 \%$ $\mathrm{CO}_{2}$ for $24 \mathrm{~h}$, media was aspirated and replaced with fresh DMEMc $(250 \mu \mathrm{L})$ at $1 \times$ $10^{6} \mathrm{vg} / \mathrm{cell}$ of PHP.eB and Tz-PHP.eB. Cells were then incubated at $37^{\circ} \mathrm{C}$ in a $5 \%$ $\mathrm{CO}_{2}$ chamber for $24 \mathrm{~h}$ in virus-loaded media, and fluorescent microscopy images (Fig. 2a) were acquired before aspiration and replacement with $500 \mu \mathrm{L}$ fresh DMEMc. Cells were cultured for one additional day prior to collection. Media was aspirated, and PBS $(300 \mu \mathrm{L})$ was added. Cells were trypsinized and transferred to $1.5 \mathrm{~mL}$ centrifuge tubes. Cells were centrifuged at $1500 \mathrm{rpm}$ for $3 \mathrm{~min}$ at room temperature and then washed with $1 \mathrm{x}$ PBS. This cell washing cycle was repeated two times. In all, $4 \%$ paraformaldehyde (PFA) was added, and the pelleted cells were further kept for $10 \mathrm{~min}$ at room temperature to fix the cells. Fixed cells were centrifuged and PFA solution removed. Cells were washed with 1x PBS $(500 \mu \mathrm{L})$, centrifuged, and supernatant removed. This washing cycle was repeated two times. After cells were resuspended in $1 \mathrm{x}$ PBS $(500 \mu \mathrm{L})$ and transferred to $5 \mathrm{~mL}$ flow cytometer tubes on ice, the frequency of green fluorescent cells was analyzed using a flow cytometer (BD FACScan with $488 \mathrm{~nm}$ excitation laser) and FlowJo v10.1 software (TreeStar) (Fig. 2b). Methods required for the subsequent evaluation of (NOTA) $)_{8}$-PHP.eB transduction (Supplementary Fig. 4) are provided in the Supplementary Methods.

\section{In vitro evaluation of AAV9 transduction before and after surface amine} modification with Tz-NHS ester. AAV9:CAG-mNeonGreen $\left(1 \times 10^{13} \mathrm{vg}, 17 \mathrm{pmol}\right)$ in 1x PBS ( $440 \mu \mathrm{L}, \mathrm{pH}$ adjusted to 8 with $0.1 \mathrm{M} \mathrm{Na}_{2} \mathrm{CO}_{3}$ solution (pH 9.1)), $10 \mathrm{mM}$ tetrazine- PEG $_{5}$-NHS $(1 \mu \mathrm{L}, 10 \mathrm{nmol})$ in anhydrous DMSO was added. After reaction and dialysis in 1xPBS, transduction of CAG-mNeonGreen by Tz-AAV9 and AAV9 (Fig. 2a, b) was compared in HEK293T cells using the same procedure as above.

In vitro evaluation of AAV9-TC transduction before and after tetracysteine modification with TCEP. AAV9-TC:CAG-mNeonGreen samples $\left(5.8 \times 10^{12} \mathrm{vg}, 10\right.$ pmol) in 1x PBS $(100 \mu \mathrm{L})$ were incubated for $30 \mathrm{~min}$ at room temperature after the addition of $5 \mathrm{mM}$ TCEP $(5 \mathrm{nmol}, 1 \mu \mathrm{L})$. The reaction mixture was dialyzed in a mini-dialysis device (20 kDa molecular weight cut-off (MWCO)) in $500 \mathrm{~mL} 1 \mathrm{x}$ PBS overnight at $4{ }^{\circ} \mathrm{C}$. Transduction of CAG-mNeonGreen by HS-AAV9-TC and AAV9-TC (Fig. 2a, b) was compared in HEK293T cells using the same procedure as above. Methods required for the subsequent evaluation of (NOTA) ${ }_{8}$-AAV9-TC transduction (Supplementary Fig. 5) are provided in the Supplementary Methods.

Sodium dodecyl sulfate-polyacrylamide gel electrophoresis of AAVs. AAVs used for in vitro transduction and in vivo PET studies were concentrated to $20 \mu \mathrm{L}$ and $150 \mu \mathrm{L}$ volume, respectively, using a spin filter (MWCO 100k). AAV capsids $(5-10 \mu \mathrm{L})$ were denatured in tris-glycine Sodium dodecyl sulfate (SDS) sample buffer (ThermoFisher Scientific, LC2676) and treated for $2 \mathrm{~min}$ at $85^{\circ} \mathrm{C}$. This solution was loaded into and separated on a $14 \%$ tris-glycine mini gel (ThermoFisher Scientific, XP00140BOX) under $225 \mathrm{~V}$ for $45 \mathrm{~min}$ in an electrophoresis chamber filled with tris-glycine SDS running buffer (ThermoFisher Scientific, LC2675). Sharp pre-stained protein standard ladder protein images (ThermoFisher Scientific, LC5800) were loaded to compare the molecular weight of viral proteins. The gel was washed with double distilled water, stained with Coomassie staining solution (ThermoFisher Scientific, LC6060) and imaged using an iPhone 6 cellular phone camera (Fig. 2e, Supplementary Figs. $4 \mathrm{~b}$ and $5 \mathrm{~b}$ ). The gels loaded with radiolabeled AAVs were further exposed on a phosphor screen in a cassette (Molecular Dynamics, CA) overnight, which was then scanned using the Phosphorimager (Amersham Bio-Science, NJ). For polyacrylamide gel electrophoresis (PAGE) of AAVs in Supplementary Figs. $4 \mathrm{~b}$ and $5 \mathrm{~b}$, the same procedure was performed without gel autoradiography.

In vivo transduction assay with ${ }^{63} \mathrm{Cu}-\mathrm{PHP} . \mathrm{eB}$ and PHP.eB. The synthesis of $\mathrm{Cu}-$ PHP.eB was performed by the following procedure. Tetrazine- $\mathrm{PEG}_{5}-\mathrm{NHS}$ in anhydrous DMSO $(1 \mu \mathrm{L}, 0.5 \mathrm{mM})$ was added to PHP.eB:CAG-GFP $\left(2.1 \times 10^{12} \mathrm{vg}\right.$, $3.5 \mathrm{pmol}$ ) in 1x PBS $\left(180 \mu \mathrm{L}, \mathrm{pH}\right.$ adjusted to 8 with $0.1 \mathrm{M} \mathrm{Na}_{2} \mathrm{CO}_{3}$ solution (pH 9.1)). After incubation for $1 \mathrm{~h}$ at room temperature, the reaction mixture was dialyzed in a mini-dialysis device ( $20 \mathrm{kDa}$ molecular weight cut-off (MWCO)) at 4 ${ }^{\circ} \mathrm{C}$ overnight. The dialyzed solution was incubated with copper-incorporated (NOTA) $)_{8}$-TCO $(5 \mu \mathrm{L}, 10 \mu \mathrm{M})$ for $30 \mathrm{~min}$ and then diluted to $15 \mathrm{~mL}$ with PBS. The solution was concentrated using a centrifugal filter unit (MWCO 100k, 3000 $\times g$ for $10 \mathrm{~min}$ ). Dilution in $15 \mathrm{~mL}$ PBS and concentration was repeated twice. The size of the isolated ${ }^{63} \mathrm{Cu}-\mathrm{PHP} . \mathrm{eB}$ and non-labeled PHP.eB was measured using a Zetasizer (Malvern). After titration of the ${ }^{63} \mathrm{Cu}-\mathrm{PHP} . \mathrm{eB}$ and the non-labeled PHP.eB solution, $1.5 \times 10^{10} \mathrm{vg}$ of ${ }^{63} \mathrm{Cu}$-PHP.eB:CAG-GFP or PHP.eB:CAG-GFP as a control were injected in C57BL/6 mice ( $n=2$ per group). After three weeks, mice were euthanized by Euthosol under deep isoflurane and perfused with DMEM followed by $4 \%$ paraformaldehyde (in PBS). The harvested brains were further fixed in $4 \%$ paraformaldehyde (PFA) in PBS for a day. Fluorescent images of the sliced brain were aquired and quantified (Fig. 2 c, d).

Radiolabeling of AAVs. All radiolabeling experiments were conducted under a radiation use authorization approved by the University of California, Davis (Davis, CA) and Stanford University (Palo Alto, CA). Molar activity in Fig. 1 is calculated from the average molar activity described in the quality control report from the Washington University, School of Medicine MIR cyclotron facility (the source of Cu-64 used in this study). For radiolabeling of AAV9 and PHP.eB, pH of AAV9: CAG-mNeonGreen $\left(1 \times 10^{13} \mathrm{vg}, 16 \mathrm{pmol}\right.$ in $0.4 \mathrm{~mL}$ PBS $)$ and PHP.eB:CAG-GFP $\left(4.2 \times 10^{12} \mathrm{vg}, 7 \mathrm{pmol}\right.$ in $0.2 \mathrm{~mL}$ PBS$)$ was adjusted to 8 with $0.1 \mathrm{M} \mathrm{Na}_{2} \mathrm{CO}_{3}$ solution ( $\mathrm{pH}$ 9.2), and $1 \mathrm{mM}$ tetrazine- $\mathrm{PEG}_{5}-\mathrm{NHS}(1 \mathrm{nmol}, 1 \mu \mathrm{L})$ in anhydrous DMSO was added to the AAV solutions. The reaction mixtures were incubated at $4{ }^{\circ} \mathrm{C}$ for $2 \mathrm{~h}$, transferred to a mini-dialysis device $(20 \mathrm{kDa}$ molecular weight cut-off (MWCO)), and dialyzed in $1 \mathrm{x}$ PBS $(0.5 \mathrm{~L})$ overnight. Tetrazine-conjugated AAVs (Tz-AAVs) were further reacted with ${ }^{64} \mathrm{Cu}$-chelated (NOTA) ${ }_{8}$-TCO for $30 \mathrm{~min}$, freshly prepared from a reaction of ${ }^{64} \mathrm{CuCl}_{2}(37-74 \mathrm{MBq}(1-2 \mathrm{mCi}), 2-3 \mu \mathrm{L})$ and $10 \mu \mathrm{M}(\mathrm{NOTA})_{8}$-TCO $(20 \mathrm{pmol}, 2 \mu \mathrm{L})$ in ammonium citrate buffer $(10 \mu \mathrm{L}, \mathrm{pH} 6.5)$. The incorporation of $\mathrm{Cu}-64$ to (NOTA) $)_{8}$-TCO was monitored by instant thin-layer chromatography and completed in $30 \mathrm{~min}$. Radiolabeled AAVs $\left({ }^{64} \mathrm{Cu}-\mathrm{AAV} 9\right.$ and -PHP.eB) were purified by size-exclusion chromatography (bed volume: $3 \mathrm{~mL}$ ) or centrifugal filter unit (MWCO 100k, $3000 \mathrm{~g}$ for $10 \mathrm{~min}$ ) with three cycles of dilution with PBS $(15 \mathrm{~mL})$ and concentrated in $\sim 200 \mu \mathrm{L}$ volume. For radiolabeling of AAV9-TC, AAV9-TC:CAG-mNeonGreen $\left(3 \times 10^{13} \mathrm{vg}, 50 \mathrm{pmol}\right.$, in $0.1 \mathrm{~mL}$ PBS) was treated with $5 \mathrm{mM}$ tris $(2$-carboxyethyl)phosphine (TCEP) $(5 \mathrm{nmol}, 1 \mu \mathrm{L})$ for $30 \mathrm{~min} .{ }^{64} \mathrm{Cu}$-chelated (NOTA) ${ }_{8}$-MI, obtained from the incorporation of ${ }^{64} \mathrm{CuCl}_{2}$ (74 MBq $(2 \mathrm{mCi}), 2.5 \mu \mathrm{L})$ to $10 \mu \mathrm{M}$ (NOTA) $)_{8}$-MI $(35 \mathrm{pmol}, 3.5 \mu \mathrm{L})$ in ammonium citrate buffer $(10 \mu \mathrm{L}, \mathrm{pH} 6.5)$, was added to disulfide-reduced SH-AAV9-TC and then the $\mathrm{pH}$ adjusted to 7-7.5 with $0.1 \mathrm{M} \mathrm{NaOH}$. After incubation at room temperature for $1 \mathrm{~h},{ }^{64} \mathrm{Cu}$-AAV9-TC was isolated by size-exclusion chromatography and concentrated in $\sim 200 \mu \mathrm{L}$ volume.

PHP.eB and AAV9 labeling with (NOTA) ${ }_{8}$-A555-TCO for the characterization of the number of labels per AAV particle. PHP.eB:CAG-mNeonGreen $(1.2 \times$ $\left.10^{12} \mathrm{vg}, 2 \mathrm{pmol}\right)$ or AA9:CAG-mNeonGreen $\left(2 \times 10^{12} \mathrm{vg}, 3.3 \mathrm{pmol}\right)$ in PBS buffer $(\mathrm{pH} 8,0.1 \mathrm{~mL})$ was reacted with tetrazine- $\mathrm{PEG}_{5}-\mathrm{NHS}(0.4-0.7$ and $0.7-1$ equivalents, respectively) at room temperature for $30 \mathrm{~min}$. After addition of $0.1 \mathrm{~mL}$ PBS, the reaction mixture was dialyzed (MWCO 20k) in PBS buffer three times $(15 \mathrm{~mL}$ each dialysis for $2 \mathrm{~h}$ and $50 \mathrm{~mL}$ overnight). Dialyzed Tz-PHP.eB or -AAV9 mixtures were reacted with (NOTA) $)_{8}$-A555-TCO (20-40 and 30-50 pmol, respectively) for $30 \mathrm{~min}$ at room temperature. Reaction mixture was diluted to $15 \mathrm{~mL}$ with PBS and filtered in a centrifugal filter unit (MWCO $100 \mathrm{k}, 3000 \times \mathrm{g}$ for $10 \mathrm{~min}$ ). To the concentrated product in PBS $(\sim 0.15 \mathrm{~mL})$, PBS $(15 \mathrm{~mL})$ was added and centrifuged (MWCO 100k, 3000 $\times g$ for $10 \mathrm{~min})$. The concentrated solution $(\sim 0.15 \mathrm{~mL})$ was transferred to a centrifugal tube, and the volume was adjusted to $0.2 \mathrm{~mL}$. The number of AAVs and labels were then measured (Supplementary Table 3).

AAV9-TC labeling with A555-C2-MI for the characterization of the number of labels per AAV particle. AAV9-TC:CAG-mNeonGreen $\left(1.7 \times 10^{12} \mathrm{vg}, 3 \mathrm{pmol}\right)$ in PBS (pH 7, 0.1 mL) was treated with $0.1 \mathrm{mM}$ tris(2-carboxyethyl)phosphine (TCEP) $(0.3 \mathrm{nmol})$ for $30 \mathrm{~min}$. Alexa555-C2-MI $(60 \mathrm{pmol})$ in dissolved in anhydrous DMSO was added to a reduced form of AAV9-TC (SH-AAV9-TC). After incubation at room temperature for $1 \mathrm{~h}$, the reaction mixture was diluted with 15 $\mathrm{mL}$ PBS and filtered in a centrifugal filter unit (MWCO 100k, 3000 $\times \mathrm{g}$ for $10 \mathrm{~min}$ ) To a concentrated product in PBS $(\sim 0.15 \mathrm{~mL})$, PBS was added again and centrifuged (MWCO $100 \mathrm{k}, 3000 \times g$ for $10 \mathrm{~min}$ ). Concentrated solution $(\sim 0.15 \mathrm{~mL}$ ) was transferred to a centrifugal tube, and the volume was adjusted to $0.2 \mathrm{~mL}$. The number of AAVs and labels were then measured. The resulting optical label is denoted as A555 at Supplementary Table 3.

PHP.eB and AAV9 labeling with A555 for in vivo optical imaging. PPHP.eB: CAG-DIO-GFP and AAV9:CAG-mNeonGreen was directly labeled with Axexa555NHS ester. In brief, $1 \mathrm{mM}$ Alexa555-NHS ( $2 \mu \mathrm{L}$ in DMSO, $2 \mathrm{nmol})$ was added to PHP.eB $\left(7 \times 10^{12} \mathrm{vg}, 12 \mathrm{pmol}\right)$ or AA9 $\left(5.2 \times 10^{12} \mathrm{vg}, 7 \mathrm{pmol}\right)$ in PBS buffer $(\mathrm{pH} 8)$ After $1 \mathrm{~h}$, the mixture was transferred to a mini-dialysis device $(20 \mathrm{kDa}$ molecular weight cut-off (MWCO) and dialyzed in 1xPBS $(0.5 \mathrm{~L})$ overnight. The dialyzed solution was diluted with $15 \mathrm{~mL}$ of PBS solution and filtered in a centrifugal filter unit (MWCO 100k, 3000 $\times g$ for $10 \mathrm{~min}$ ) with three cycles of dilution with PBS (15 $\mathrm{mL}$ ). This solution was reformulated with 1x PBS before the in vivo study shown in Supplementary Fig. 9. The resulting label is denoted as A555 in Supplementary Figs. $7 \mathrm{a}$ and 9.

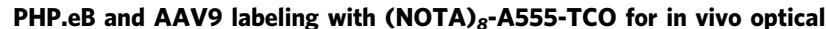
imaging. PHP.eB:CAG-DIO-GFP and AAV9:CAG-mNeonGreen was labeled with tetrazine- $\mathrm{PEG}_{5}-\mathrm{NHS}$ using the same procedure as above. In brief, $1 \mathrm{mM}$ tetrazine- 
PEG $_{5}$-NHS $(2 \mu \mathrm{L}$ in DMSO, $2 \mathrm{nmol})$ was added to PHP.eB $\left(1.4 \times 10^{13} \mathrm{vg}, 23 \mathrm{pmol}\right)$ in PBS buffer ( $\mathrm{pH} 8)$. After $1 \mathrm{~h}$, the mixture was transferred to a mini-dialysis device (20 kDa molecular weight cut-off (MWCO)) and dialyzed in 1x PBS (0.5 L) overnight. Tz-PHP.eB solution was reacted with $0.1 \mathrm{mM}(\mathrm{NOTA})_{8}$-A555-TCO (1 $\mu \mathrm{L}, 0.1 \mathrm{nmol}$ ) for $30 \mathrm{~min}$ at room temperature. The reaction mixture was diluted to $15 \mathrm{~mL}$ PBS solution and filtered in a centrifugal filter unit (MWCO 100k, $3000 \mathrm{~g}$ for $10 \mathrm{~min}$ ) with three cycles of dilution with PBS $(15 \mathrm{~mL})$. Concentrated (NOTA $)_{8^{-}}$ A555-PHP.eB was formulated with 1x PBS for the in vivo study shown in Fig. $3 \mathrm{i}$ and Supplementary Fig. 10.

Animal models. All animal experiments were conducted under an animal use protocol approved by the University of California, Davis, Institutional Animal Care and Use Committee (IACUC) or Stanford University, Administrative Panel on Laboratory Animal Care (APLAC). For PET studies and biodistribution, ${ }^{64} \mathrm{Cu}-$ labeled AAVs were evaluated in wild-type 7-9-week-old female C57BL/6 and 9 week-old-female BALB/c mice (The Jackson laboratory, Bar Harbor, ME). For neuraminidase-treated $B A L B / c$ mice, neuraminidase $(0.12$ units $/ 20 \mu \mathrm{L}$, SigmaAldrich, \#N7885) was intranasally administered $3 \mathrm{~h}$ before the injection of ${ }^{64} \mathrm{Cu}-$ AAVs. The classical pharmacokinetics and biodistribution studies with qPCR were conducted with wild-type 5-6 week-old female C57BL/6 mice (Charles River).

PET/CT scans and biodistribution. Mice were anesthetized with $3.0 \%$ isoflurane in oxygen and maintained under $1.5-2.0 \%$ isoflurane. ${ }^{64} \mathrm{Cu}$-AAV9 injections in C57BL/6 mice $(n=3,421 \pm 25 \mathrm{KBq}),{ }^{64} \mathrm{Cu}-\mathrm{AAV} 9-\mathrm{TCO}$ injections in C57BL/6 mice $(n=3,760 \pm 156 \mathrm{KBq}),{ }^{64} \mathrm{Cu}-\mathrm{PHP} . \mathrm{eB}$ injections in C57BL/6 mice $(n=3,628$ $\pm 581 \mathrm{KBq}), \mathrm{BALB} / \mathrm{c}$ mice $(n=3,477 \pm 19 \mathrm{KBq})$, or $\mathrm{BALB} / \mathrm{c}$ mice treated with neuraminidase $(n=3,718 \pm 30 . \mathrm{KBq})$ were administered via the tail vein on an Inveon DPET small animal PET scanner (Siemens Medical Solutions USA,

Knoxville, TN). Animals were scanned for $30 \mathrm{~min}$ at 0,4 , and $21 \mathrm{~h}$ post injection. After each PET scan, the animals were moved to a small animal Inveon MM CT system (Siemens Medical Solutions USA, Knoxville, TN) and a CT scan was conducted to obtain anatomical information for co-registration of PET/CT images. After the final imaging time point, mice were euthanized by Euthosol under deep isoflurane. Animals were then perfused with DMEM solution. Blood, heart, lungs, liver, spleen, kidneys, stomach, intestine, muscle, bone, and brain were harvested for biodistribution analysis. Radioactivity in each organ was measured with a 1470 automatic gamma counter (PerkinElmer, CT) after which organ weights were taken on a balance. Biodistribution of AAVs was presented as percent injected dose per gram (\% ID/g). In some cases, brains were sectioned at $2 \mathrm{~mm}$ thickness to obtain ex vivo autoradiography.

ROI analysis and time-activity curves. All PET images were reconstructed with the maximum a posteriori (MAP) reconstruction algorithm in Inveon Acquisition Workspace (Siemens Medical Solutions Inc., USA) and analyzed with Inveon Research Workspace 4.2 (Siemens Medical Solutions Inc., USA) after the coregistration of PET/CT images. Regions of interest (ROIs) were drawn in the heart chamber for blood, whole brain, and liver. The time-activity curves (TAC) of blood, brain, and liver radioactivities at 0,4 , and $21 \mathrm{~h}$ were analyzed with Prism 8 (Graphpad). Blood TACs were fitted with one phase decay. Radioactivity density from image analysis is presented as percent injected dose per cubic centimeter (\% $\mathrm{ID} / \mathrm{cc})$.

Early brain accumulation and Logan analysis of ${ }^{64} \mathrm{Cu}$-AAVs. Initial PET data from the $30 \mathrm{~min}$ scan was segmented into six frames $(5 \mathrm{~min} / \mathrm{frame})$. Blood radioactivity in the brain $(\% \mathrm{ID} / \mathrm{cc})$ over time was calculated by multiplying the brain radioactivity by the brain vascular volume (8\%) (estimated by the ratio of brain and blood radioactivity of AAV9 and AAV9-TC in C57BL/6 and PHP.eB in BALB/c at 5 min after injection). The blood radioactivity over time was then subtracted from total brain activity $(\% \mathrm{ID} / \mathrm{ccc})$ to estimate the brain accumulation. A Logan plot (Fig. 3h) was then applied for the calculation of the uptake rate of each AAV in the brain at a given time after the administration of $\mathrm{AAVs}^{17} . \mathrm{C}_{\mathrm{B}}(\mathrm{t})$ and $\mathrm{C}_{\mathrm{T}}(\mathrm{t})$ are the radioactivity concentration in the blood and target at a given time, and $\operatorname{Int}_{\mathrm{B}}(\mathrm{t})$ and $\operatorname{Int}_{\mathrm{T}}(\mathrm{t})$ are the accumulated radioactivity in the blood and target, respectively, from the time of injection to time $(t)$. $\operatorname{Int}_{T}(t) / C_{T}(t)$ vs $\operatorname{IntC}_{B}(t) / C_{T}(t)$ was then plotted.

Confocal microscopy. For imaging of fluorescent AAVs (Fig. 3i and Supplementary Fig. 9), mice were anesthetized with $3.0 \%$ isoflurane in oxygen and maintained under $1.5-2.0 \%$ isoflurane. A555-PHP.eB $\left(0.1 \mathrm{~mL}, 1 \times 10^{11} \mathrm{vg}\right)$ or $(\text { NOTA })_{8}$-A555-PHP.eB $\left(0.1 \mathrm{~mL}, 2 \times 10^{10} \mathrm{vg}\right)$ or was administered to C57BL/6 mice ( $n=14$ total) through tail vein injection. Mice were euthanized at 4, 24 and $48 \mathrm{~h}$ by Euthosol under deep isoflurane, and perfused with DMEM solution followed by $4 \%$ PFA in PBS at pH 7. To stain the brain endothelium, FITC-lectin (25 $\mu \mathrm{L}, 50 \mu \mathrm{g})$ was injected via the tail-vein $15 \mathrm{~min}$ before euthanization. Brains were collected and kept in 4\% PFA overnight. Brain tissue was then sliced to $100 \mu \mathrm{m}$ thick sections on a vibratome (Leica VT-1000S) in PBS and mounted onto microscope slides. Fluorescence images of AAV distribution on the endothelium were acquired with a confocal microscope (Zeiss AxioObserver), a $\times 40$ objective,
488 and $561 \mathrm{~nm}$ lasers, and HQ camera. The microscope was controlled by Slidebook software (Intelligent Imaging Innovations).

Brain slices for in vivo transduction (Figs. $2 \mathrm{c}$ and $3 \mathrm{~g}$ ) were mounted on microscope slides and imaged using a confocal microscope (Leica DMi8) controlled with LAS X software. All images were recorded at the same laser power and gain control. Images were acquired with a $10 \mathrm{x}$ lens and fluorescence images were analyzed using ImageJ.

Synthesis of PEG(40 kDa)-AAV9 for cryo-electron microscopy. To a solution $(0.4 \mathrm{~mL}, \mathrm{PBS})$ of PEG $(40 \mathrm{kDa})-\mathrm{NH}_{2}(2.5 \mathrm{mg}, 62 \mathrm{nmol})$ was added TCO-PEG ${ }_{4}-\mathrm{NHS}$ ester $(0.5 \mathrm{mg}, 0.86 \mu \mathrm{mol})$. The $\mathrm{pH}$ was adjusted to 8.6 with $0.1 \mathrm{M}$ sodium carbonate ( $\mathrm{pH}$ 9.2). After a $2 \mathrm{~h}$ reaction at room temperature, the reaction mixture was diluted with $1 \mathrm{~mL}$ deionized water then dialyzed overnight with 10k MWCO and the purified solution was transferred to a cryotube and freeze-dried (Supplementary Fig. 7b). PEG(40 kDa)-AAV9 was obtained as illustrated in Supplementary Fig. 7a. Tz-AAV9 was obtained from a reaction with AA9:CAG-mNeonGreen $\left(5.9 \times 10^{12} \mathrm{vg}, 9.8 \mathrm{pmol}\right)$ and tetrazine- $\mathrm{PEG}_{5}-\mathrm{NHS}(2 \mu \mathrm{L}$ in DMSO, $2 \mathrm{nmol})$ under the same procedure as in Optical labeling of AAVs and was reacted with $10 \mu \mathrm{M}$ PEG ( $40 \mathrm{kDa})$-TCO $(4 \mu \mathrm{L}, 40 \mathrm{pmol})$ for $30 \mathrm{~min}$ at room temperature. The reaction mixture was diluted to $15 \mathrm{~mL}$ PBS solution and filtered in a centrifugal filter unit (MWCO 100k, $3000 \mathrm{~g}$ for $10 \mathrm{~min}$ ) with two cycles of dilution with PBS $(15 \mathrm{~mL})$. PEG (40 kDa)-AAV9 was further concentrated to $40 \mu \mathrm{L}$ volume for cryo-EM.

Cryo-electron microscopy of PEG(40 kDa)-AAV9. Cryo-Electron microscopy collection was performed on a Glacios ${ }^{\mathrm{mit}}$ Cryo-TEM operating with a field-emission gun at $200 \mathrm{kV}$. Cryo-EM grid was prepared with a $30 \mu \mathrm{L}$ solution containing PEG ( $40 \mathrm{kDa}$ )-AAV9, which was placed on Quantifoil R1/2 Cu 300 mesh grids. The grids were pretreated with $10 \mathrm{mAmp}$ of glow discharge for 40 seconds. After $1 \mathrm{~min}$ of on-grid incubation the excess solution was removed and quickly plunged into liquid ethane using an FEI Vitrobot Mark III semi-automated cryo-plunger. The PEG(40 kDa)-AAV9 particles were embedded into a thin layer of vitrified ice and transferred into the imaging chamber using a Gatan 626 cryo-transferring system. The grids were examined at $\times 50,000$ magnification and images were captured using a Gatan K3. The cryoEM images were inverted in contrast to illustrate the positive density of the molecular mass against the dark ice background. The digital images were recorded with a pixel size of $0.85 \AA$ using autofocusing scripts in the SerialEM package set to a defocus range of 0.5-2.0 micron. The digital images with minimum stigmatism or drift were selected for further statistical analysis and figure preparation. Corresponding EM images are shown in Fig. $2 \mathrm{~g}$.

Biodistribution and pharmacokinetics of AAVs with qPCR. For the biodistribution study in Fig. 3f, g, PHP.eB $\left(7.4 \times 10^{10} \mathrm{vg}, n=4\right)$ and (NOTA) $)_{8}$-A555PHP.eB $\left(1.4 \times 10^{11} \mathrm{vg}, n=4\right)$ in PBS $(0.1 \mathrm{~mL})$ were injected through the mouse $(\mathrm{C} 57 \mathrm{BL} / 6)$ tail vein. At $21 \mathrm{~h}$ after injection, mice were euthanized by Euthosol under deep isoflurane, and then perfused with DMEM. Heart, lungs, liver, spleen, kidneys, brain, and blood were harvested as 3-4 fractions per tissue and 100-200 $\mu \mathrm{L}$ blood per mouse. Collected tissues were immediately frozen in $2 \mathrm{~mL}$ cryotubes under liquid nitrogen and stored at $-80{ }^{\circ} \mathrm{C}$. Viral vector and mouse genomic DNAs were extracted from tissues by a DNeasy Blood and Tissue Kit (Qiagen) according to the kit protocol and quantified by quantitative polymerase chain reaction ( $\mathrm{qPCR}$ ) with a TaqMan assay. The primers and TaqMan probe used for the WPRE sequence were 5'-GCATTGCCACCACCTGTCA (forward) and 5'TCCGCCGTGGCAATAGG (reverse), and 5'-CTTTCCGGGACTTTCG (FAM). The primers and TaqMan probe targeting the mouse glucagon gene as a housekeeping gene were $5^{\prime}$-GTTCTCTCTGTATTGTCCTTTCAAAGTCT (forward) and 5'-CAAAGTCCCTGAAGGTTCTGAGATG (reverse), and 5'-CCCTGGTC ATGTTTTTAA (FAM). Quantification was performed by a standard curve from a serial dilution of known standard plasmid concentration. For the classical PK study of blood clearance, PHP.eB $\left(1.35 \times 10^{11} \mathrm{vg}, n=4\right)$ and (NOTA) ${ }_{8}$-A555-PHP.eB $\left(1.17 \times 10^{11} \mathrm{vg}, n=4\right)$ in PBS $(0.1 \mathrm{~mL})$ were injected through the mouse $(\mathrm{C} 57 \mathrm{BL} / 6)$ tail vein. Blood $(\sim 100-200 \mu \mathrm{L})$ was collected from the mouse orbital sinus at $0.5,4$ and $21 \mathrm{~h}$ after administration to a Microtrainer containing EDTA (BD science, $\mathrm{NJ}$ ). Collected blood was immediately transferred to $2 \mathrm{~mL}$ cryotubes (Sarstedt, Germany) and further frozen in liquid nitrogen. The viral vectors from $100 \mu \mathrm{L}$ of blood were extracted by DNeasy Blood and Tissue Kit (Qiagen) according to the kit protocol and quantified by the TaqMan assay targeting the WPRE sequence. qPCR was performed with QuantStudio6 Flex (Applied Biosystems) and CFX96 Touch real-time PCR detection system (Bio-Rad).

Image processing and data analysis. Microscopic image process and ROI analyses were performed using ImageJ, LAS X (Leica) and SlidBook6 (3i). For PET/CT data and image processing, Inveon Acquisition Workspace (Siemens) and Inveon Research Workspace 4.2 (Siemens) was used, respectively. Microsoft Excel (ver. 16.35), and GraphPad Prism 8 for macOS were used for general data and statistical analysis. PyMOL 2.0 (Molecular Graphics System) was used to process the structure of capsid. FlowJo v10.1 (Treestar) was used for data analyses of results from flow cytometry. Gating/sorting strategy is presented at Supplementary Fig. 11 and reporting summary. 


\section{Statistics and reproducibility}

All statistical analyses were performed in GraphPad Prism software (Prism 8.0). The statistical tests with confidence intervals, effect sizes, degree of freedom and $P$ values can be found in the source data. Sample size for each experiment is shown in the appropriate caption and the biological replicates across experiments are summarized below. Cell experiments in Fig. 2a, b were repeated 4, 3, and 1 times for PHP.eB, AAV9 and AAV9-TC. For Fig. 2c, fluorescent images were selected from multiple mouse brain slices obtained in 2 experiments. For Fig. $2 g$, the cryoEM images result from a single experiment. For Fig. 3a-e and G-H, PET/CT and autoradiography result from two experiments. For Fig. 3f, qPCR results were obtained from one set of experiments. For Fig. 3i, representative fluorescence images with the multichelator AAV were chosen from mice euthanized at 4 (two experiments), 24 (one experiment) and $48 \mathrm{~h}$ (one experiment). For Fig. 4, the data result from one series of experiments.

Reporting summary. Further information on research design is available in the Nature Research Reporting Summary linked to this article.

\section{Data availability}

The raw data files from mass spectrometer were processed using Byonic v 2.14.27 (Protein Metrics, San Carlos, CA) to identify peptides and subsequently infer proteins using the Mus musculus database from the Universal Protein Resource (UniProt, http:// www.uniprot.org) along with the sequences of capsid proteins. Protein Data Bank (PDB ID:3Ux1) was used to display capsid structure. Analyzed viral protein sequence data are available in the source data. The authors declare that image and quantitative data supporting the findings of this study are available within the paper and the source data and supplementary files. The raw PET images and associated data that support the findings of this study are available from the corresponding author upon reasonable request.

Received: 31 August 2019; Accepted: 31 March 2020;

Published online: 30 April 2020

\section{References}

1. Pardridge, W. Targeted delivery of protein and gene medicines through the blood-brain barrier. Clin. Pharmacol. Ther. 97, 347-361 (2015)

2. Bedbrook, C. N., Deverman, B. E. \& Gradinaru, V. Viral strategies for targeting the central and peripheral nervous systems. Annu. Rev. Neurosci. 41, 323-348 (2018).

3. Chan, K. Y. et al. Engineered AAVs for efficient noninvasive gene delivery to the central and peripheral nervous systems. Nat. Neurosci. 20, 1172-1179 (2017).

4. Deverman, B. E. et al. Cre-dependent selection yields AAV variants for widespread gene transfer to the adult brain. Nat. Biotech. 34, 204-209 (2016).

5. Mingozzi, F. \& High, K. A. Immune responses to AAV in clinical trials. Curr. Gene Ther. 11, 321-330 (2011)

6. Naso, M. F., Tomkowicz, B., Perry, W. L. \& Strohl, W. R. Adeno-Associated Virus (AAV) as a vector for gene therapy. BioDrugs 31, 317-334 (2017).

7. Smalley, E. First AAV gene therapy poised for landmark approval. Nat. Biotechnol. 35, 998-999 (2017).

8. Ginn, S. L., Amaya, A. K., Alexander, I. E., Edelstein, M. \& Abedi, M. R. Gene therapy clinical trials worldwide to 2017: an update. J. Gene Med. 20, e3015 (2018).

9. Thakore, P. I. et al. RNA-guided transcriptional silencing in vivo with S. aureus CRISPR-Cas9 repressors. Nat. Commun. 9, 1674 (2018).

10. Huang, Q. et al. Delivering genes across the blood-brain barrier: LY6A, a novel cellular receptor for AAV-PHP.B capsids. PLoS One 14, e0225206 (2019).

11. Hordeaux, J. et al. The GPI-linked protein LY6A drives AAV-PHP.B transport across the blood-brain barrier. Mol. Ther. 27, 912-921 (2019).

12. Xianxing $\mathrm{Xu}$, Yang, Jingwen, Cheng, Y. Pharmacokinetic Study of Viral Vectors for Gene Therapy: Progress and Challenges. (InTech, 2011).

13. de Kok, T. M. \& van Maanen, J. M. Evaluation of fecal mutagenicity and colorectal cancer risk. Mutat. Res. 463, 53-101 (2000).

14. Mingozzi, F. \& High, K. A. Overcoming the host immune response to adenoassociated virus gene delivery vectors: the race between clearance, tolerance, neutralization, and escape. Annu Rev. Virol. 4, 511-534 (2017).

15. Mingozzi, F. \& High, K. A. Immune responses to AAV vectors: overcoming barriers to successful gene therapy. Blood 122, 23-36 (2013).
16. Rueda, F. \& Cruz, L. J. Targeting the brain with nanomedicine. Curr. Pharm Des. 23, 1879-1896 (2017).

17. Logan, J. Graphical analysis of PET data applied to reversible and irreversible tracers. Nucl. Med. Biol. 27, 661-670 (2000)

18. Gunn, R. N., Slifstein, M., Searle, G. E. \& Price, J. C. Quantitative imaging of protein targets in the human brain with PET. Phys. Med. Biol. 60, R363-R411 (2015).

19. Bartlett, J. S. \& Samulski, R. J. Fluorescent viral vectors: a new technique for the pharmacological analysis of gene therapy. Nat. Med 4, 635-637 (1998).

20. Leopold, P. L. et al. Fluorescent virions: dynamic tracking of the pathway of adenoviral gene transfer vectors in living cells. Hum. Gene Ther. 9, 367-378 (1998).

21. Sanlioglu, S. et al. Endocytosis and nuclear trafficking of adeno-associated virus type 2 are controlled by Rac1 and phosphatidylinositol-3 kinase activation. J. Virol. 74, 9184-9196 (2000).

22. Xiao, W., Warrington, K. H., Hearing, P., Hughes, J. \& Muzyczka, N. Adenovirus-facilitated nuclear translocation of adeno-associated virus type 2 . J. Virol. 76, 11505-11517 (2002).

23. Liu, Y. et al. Site-specific modification of adeno-associated viruses via a genetically engineered aldehyde tag. Small 9, 421-429 (2013).

24. Chandran, J. S. et al. Site specific modification of adeno-associated virus enables both fluorescent imaging of viral particles and characterization of the capsid interactome. Sci. Rep. 7, 14766 (2017).

25. Lee, G. K., Maheshri, N., Kaspar, B. \& Schaffer, D. V. PEG conjugation moderately protects adeno-associated viral vectors against antibody neutralization. Biotechnol. Bioeng. 92, 24-34 (2005).

26. Yao, T. et al. Site-specific PEGylated adeno-associated viruses with increased serum stability and reduced immunogenicity. Molecules 22, 1155 (2017).

27. Stachler, M. D., Chen, I., Ting, A. Y. \& Bartlett, J. S. Site-specific modification of AAV vector particles with biophysical probes and targeting ligands using biotin ligase. Mol. Ther. 16, 1467-1473 (2008).

28. Kelemen, R. E. et al. A precise chemical strategy to alter the receptor specificity of the adeno-associated virus. Angew. Chem. Int Ed. Engl. 55, 10645-10649 (2016).

29. Horowitz, E. D., Weinberg, M. S. \& Asokan, A. Glycated AAV vectors: chemical redirection of viral tissue tropism. Bioconjug Chem. 22, 529-532 (2011).

30. DiMattia, M. A. et al. Structural insight into the unique properties of adenoassociated virus serotype 9. J. Virol. 86, 6947-6958 (2012).

31. Kothari, P. et al. Radioiodinated capsids facilitate in vivo non-invasive tracking of adeno-associated gene transfer vectors. Sci. Rep. 7, 39594 (2017)

32. Yoon, S. Y. et al. Quantitative, noninvasive, in vivo longitudinal monitoring of gene expression in the brain by co-AAV transduction with a PET reporter gene. Mol. Ther. Methods Clin. Dev. 1, 14016 (2014).

33. Kotchey, N. M. et al. A potential role of distinctively delayed blood clearance of recombinant adeno-associated virus serotype 9 in robust cardiac transduction. Mol. Ther. 19, 1079-1089 (2011).

34. van Gestel, M. A. et al. Recombinant adeno-associated virus: efficient transduction of the rat VMH and clearance from blood. PLoS One 9, e97639 (2014).

35. Liu, Y., Joo, K. I. \& Wang, P. Endocytic processing of adeno-associated virus type 8 vectors for transduction of target cells. Gene Ther. 20, 308-317 (2013).

36. Oliveira, B. L., Guo, Z. \& Bernardes, G. J. L. Inverse electron demand DielsAlder reactions in chemical biology. Chem. Soc. Rev. 46, 4895-4950 (2017).

37. Tyagarajan, K., Pretzer, E. \& Wiktorowicz, J. E. Thiol-reactive dyes for fluorescence labeling of proteomic samples. Electrophoresis 24, 2348-2358 (2003).

38. Nakashima, F. et al. Structural and functional insights into S-thiolation of human serum albumins. Sci. Rep. 8, 932 (2018).

39. Wang, G., Yan, C., Gao, S. \& Liu, Y. Surface chemistry of gold nanoparticles determines interactions with bovine serum albumin. Mater. Sci. Eng. C. 103 109856 (2019).

40. Hordeaux, J. et al. The neurotropic properties of AAV-PHP.B are limited to C57BL/6J mice. Mol. Ther. 26, 664-668 (2018).

41. Breous, E., Somanathan, S. \& Wilson, J. M. BALB/c mice show impaired hepatic tolerogenic response following AAV gene transfer to the liver. Mol. Ther. 18, 766-774 (2010).

42. Fitzpatrick, Z. et al. Influence of pre-existing anti-capsid neutralizing and binding antibodies on AAV vector transduction. Mol. Ther. Methods Clin. Dev. 9, 119-129 (2018)

43. Shen, S. et al. Terminal N-Linked galactose is the primary receptor for adenoassociated vir. J. Biol. Chem. 286, 13532-13540 (2011)

44. Bell, C. L. et al. The AAV9 receptor and its modification to improve in vivo lung gene transfer in mice. J. Clin. Invest. 121, 2427-2435 (2011).

45. Pillay, S. et al. An essential receptor for adeno-associated virus infection Nature 530, 108-112 (2016)

46. Merkel, S. F. et al. Trafficking of adeno-associated virus vectors across a model of the blood-brain barrier; a comparative study of transcytosis and transduction using primary human brain endothelial cells. J. Neurochem. 140 216-230 (2017) 
47. Pulicherla, N. et al. Engineering liver-detargeted AAV9 vectors for cardiac and musculoskeletal gene transfer. Mol. Ther. 19, 1070-1078 (2011).

48. Katrekar, D., Moreno, A. M., Chen, G., Worlikar, A. \& Mali, P. Oligonucleotide conjugated multi-functional adeno-associated viruses. Sci. Rep. 8, 1-8 (2018).

49. Haywood, T. et al. Positron emission tomography reporter gene strategy for use in the central nervous system. Proc. Natl Acad. Sci. USA 166, 11402-11407 (2019).

\section{Acknowledgements}

We thank Dr. James Wilson for the gift of AAV9, Dr. Hua Zhang for technical assistance in fluorescence microscopy of optically labeled AAVs, Dr. Soichiro Yamada for supporting confocal microscope time, the California Behavioral Health Center of Excellence Pilot Award, the Foundation for Angelman Syndrome Therapeutics, Dr. Siobhan Brady for vibratome usage and Yaping Lei and Dr. Alice Tarantal for technical advice on qPCR, Sarah Tom and Charles Smith in the Center for Molecular Genomic Imaging at UC Davis for PET/CT imaging assistance, Marina Raie for technical assistance and the CLOVER Center in the Beckman Institute at Caltech for producing AAVs. This work was supported by NIHCA112356, NIHEB028646, NIHUG3TR002866, NIFACADMCB7399-H and 17IRG33420114.

\section{Author contributions}

J.W.S. and K.W.F. designed and implemented the study, produced data, and wrote the paper with significant input from V.G., J.W.S., and L.M. performed PET imaging and analysis. B.W., S.A., P.J., and M.J., performed and commented on classical in vivo qPCR studies. M.C., E.S.I., and S.T. assessed titers, performed flow cytometry, and analyzed data. S.S. performed in vitro assays of AAVs in HEK293T cells. M.B. and R.H.C. acquired and analyzed cryoEM images. N.G., N.C.F., and T.D. provided AAVs and X.D. provided X-ray capsid images and identified critical capsid peptide sequences. K.S. and R.L. at the Stanford University Mass Spectrometry core performed viral protein mass analysis. D.S. and E.S. advised on viral vector handling and in vitro cell assays, respectively. All authors discussed the results and contributed to the completion of the paper.

\section{Competing interests}

The authors declare no competing interests.

\section{Additional information}

Supplementary information is available for this paper at https://doi.org/10.1038/s41467020-15818-4.

Correspondence and requests for materials should be addressed to K.W.F.

Peer review information Nature Communications thanks the anonymous reviewer(s) for their contribution to the peer review of this work.

Reprints and permission information is available at http://www.nature.com/reprints

Publisher's note Springer Nature remains neutral with regard to jurisdictional claims in published maps and institutional affiliations.

(c) (i) Open Access This article is licensed under a Creative Commons Attribution 4.0 International License, which permits use, sharing, adaptation, distribution and reproduction in any medium or format, as long as you give appropriate credit to the original author(s) and the source, provide a link to the Creative Commons license, and indicate if changes were made. The images or other third party material in this article are included in the article's Creative Commons license, unless indicated otherwise in a credit line to the material. If material is not included in the article's Creative Commons license and your intended use is not permitted by statutory regulation or exceeds the permitted use, you will need to obtain permission directly from the copyright holder. To view a copy of this license, visit http://creativecommons.org/ licenses/by/4.0/.

(C) The Author(s) 2020 